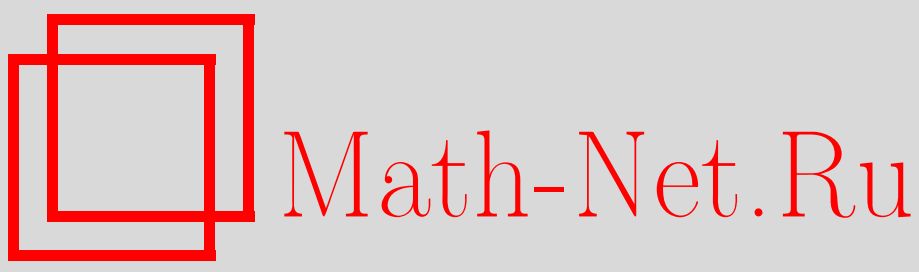

А. Ю. Колесов, Н. Х. Розов, Диффузионная буферность в одной математической модели биологии, Изв. РАН. Сер. матем., 1998, том 62, выпуск 5, 135-164

DOI: https://doi.org/10.4213/im213

Использование Общероссийского математического портала Math-Net.Ru подразумевает, что вы прочитали и согласны с пользовательским соглашением http://www . mathnet.ru/rus/agreement

Параметры загрузки:

IP: 3.93 .64 .190

26 апреля 2023 г., 13:13:01 
УДК 517.926

\author{
А. Ю. Колесов, Н. Х. Розов
}

\title{
Диффузионная буферность в одной математической модели биологии
}

\begin{abstract}
Рассматривается система дифференциально-разностных уравнений в частных производных с диффузией при граничных условиях Неймана, моделирующая одну задачу хищник-жертва. Методом бесконечномерной нормализации устанавливается феномен диффузионной буферности, т.е. существование (при подходящем выборе параметров) любого числа устойчивых пространственно неоднородных циклов.

Библиография: 16 наименований.
\end{abstract}

Будем говорить, что в параболической системе типа реакция-диффузия с граничными условиями Неймана наблюдается диффузионная буферность, если при подходящем выборе параметров такая задача имеет любое фиксированное число устойчивых пространственно неоднородных циклов. Впервые это явление с помощью численных методов обнаружено в [1] при исследовании системы двух дифференциально-разностных уравнений с диффузией (модель задачи хищник-жертва с учетом миграции хишника). В этой же работе был поставлен вопрос о теоретическом объяснении явления диффузионной буферности на модельной сингулярно возмущенной краевой задаче без запаздывания. Его решение содержится в статьях [2]-[5], а также в монографии [6]. В настоящей работе полученные в этих публикациях результаты переносятся непосредственно на краевую задачу из [1].

\section{§ 1. Постановка задачи и основные результаты}

1.1. На основе синтеза известных идей Вольтерра и Хатчинсона в [7] для моделирования взаимодействия хищника со своей жертвой предложена следующая краевая задача:

$$
\begin{gathered}
\frac{\partial N_{1}}{\partial t}=\frac{r_{1}}{1+a}\left[1+a\left(1-N_{2}\right)-N_{1}\left(t-h_{1}, x\right)\right] N_{1}, \\
\frac{\partial N_{2}}{\partial t}=D \frac{\partial^{2} N_{2}}{\partial x^{2}}+r_{2}\left[N_{1}-N_{2}\left(t-h_{2}, x\right)\right] N_{2}, \\
\left.\frac{\partial N_{2}}{\partial x}\right|_{x=0}=\left.\frac{\partial N_{2}}{\partial x}\right|_{x=1}=0 .
\end{gathered}
$$

Работа выполнена при финансовой поддержке РФФИ и при поддержке INTAS.

(C) А.Ю. Колесов, Н.Х. Розов 1998 
Здесь $N_{1}(t, x), N_{2}(t, x)$ - плотности популяций (в момент $t$ в точке $\left.x\right)$ жертвы и хишника соответственно; $r_{j}>0, h_{j}>0, j=1,2,-$ мальтузианские коэффициенты линейного роста и возрасты половозрелости; $a>0$ - коэффициент давления хишника на жертву; $D>0$ - коэффициент подвижности хишника (подвижность жертвы не учитывается). Предполагается, что среда обитания однородна как для хишника, так и для жертвы и представляет собой отрезок $0 \leqslant x \leqslant 1$.

Обозначим через $C\left(\left[-h_{j}, 0\right] ; W_{2}^{1}\right), j=1,2$, банаховы пространства непрерьвных по $s \in\left[-h_{j}, 0\right]$ в метрике $W_{2}^{1}[0,1]$ функций $\varphi(s, x)$ с нормами

$$
\|\varphi(s, x)\|=\max _{-h_{j} \leqslant s \leqslant 0}\|\varphi\|_{W_{2}^{1}}, \quad j=1,2 .
$$

Рассмотрим, далее, решение $\left(N_{1}(t, x), N_{2}(t, x)\right)$ краевой задачи (1.1)-(1.3) с произвольными неотрицательньми начальными функциями

$$
\left(\varphi_{1}(s, x), \varphi_{2}(s, x)\right)=\varphi(s, x) \in E=C\left(\left[-h_{1}, 0\right] ; W_{2}^{1}\right) \times C\left(\left[-h_{2}, 0\right] ; W_{2}^{1}\right),
$$

которое строится методом шагов.

Например, на первом шаге, т.е. при $0 \leqslant t \leqslant h_{0}=\min \left(h_{1}, h_{2}\right)$, сначала из уравнения (1.1) находим

$N_{1}(t, x)=\Pi\left(N_{2}\right)=\varphi_{1}(0, x) \exp \left\{\frac{r_{1}}{1+a} \int_{0}^{t}\left[1+a\left(1-N_{2}(s, x)\right)-\varphi_{1}\left(s-h_{1}, x\right)\right] d s\right\}$.

Подставляя затем это выражение в уравнение (1.2), для определения $N_{2}(t, x)$ получаем интегро-дифференциальную смешанную задачу

$$
\begin{gathered}
\frac{\partial N_{2}}{\partial t}=D \frac{\partial^{2} N_{2}}{\partial x^{2}}+r_{2}\left[\Pi\left(N_{2}\right)-\varphi_{2}\left(t-h_{2}, x\right)\right] N_{2}, \\
\left.\frac{\partial N_{2}}{\partial x}\right|_{x=0}=\left.\frac{\partial N_{2}}{\partial x}\right|_{x=1}=0,\left.\quad N_{2}\right|_{t=0}=\varphi_{2}(0, x),
\end{gathered}
$$

однозначно разрешимую при $0 \leqslant t \leqslant h_{0}$. Действительно, из дифференциального неравенства

$$
\begin{gathered}
\frac{\partial N_{2}}{\partial t} \leqslant D \frac{\partial^{2} N_{2}}{\partial x^{2}}+b(t, x) N_{2} \\
b(t, x)=r_{2}\left[\varphi_{1}(0, x) \exp \left\{r_{1} \int_{0}^{t}\left(1-\varphi_{1}\left(s-h_{1}, x\right) /(1+a)\right) d s\right\}-\varphi_{2}\left(t-h_{2}, x\right)\right]
\end{gathered}
$$

и принципа максимума вытекает априорная оценка

$$
0 \leqslant N_{2}(t, x) \leqslant N_{2}^{*}(t, x), \quad 0 \leqslant t \leqslant h_{0}, \quad 0 \leqslant x \leqslant 1
$$

где $N_{2}^{*}$ - решение линейной краевой задачи

$$
\frac{\partial N_{2}^{*}}{\partial t}=D \frac{\partial^{2} N_{2}^{*}}{\partial x^{2}}+b(t, x) N_{2}^{*},\left.\quad \frac{\partial N_{2}^{*}}{\partial x}\right|_{\substack{x=0 \\ x=1}}=0,\left.\quad N_{2}^{*}\right|_{t=0}=\varphi_{2}(0, x) .
$$


А отсюда и из общих свойств решений уравнений параболического типа (см. [8], [9]) следует существование и единственность при всех $0 \leqslant t \leqslant h_{0}, 0 \leqslant x \leqslant 1$ классического решения задачи (1.4). При $h_{0} \leqslant t \leqslant 2 h_{0}$ рассмотрению подлежит аналогичная (1.4) смешанная задача с начальными условиями при $t=h_{0}$ и т.д.

Из описанного способа построения решения $\left(N_{1}(t, x), N_{2}(t, x)\right)$ заключаем, что, во-первых, оно определено при всех $t \geqslant 0,0 \leqslant x \leqslant 1$, а во-вторых, формулой

$$
T[t, \varphi(s, x)]=\left(N_{1}(t+s, x), N_{2}(t+s, x)\right)
$$

в фазовом пространстве $E$ задается сильно непрерывная по $t$ полугруппа нелинейных ограниченных операторов, гладко (по Фреше) зависящая от начального условия $\varphi(s, x) \in E$ и параметров. Это в свою очередь позволяет практически без изменений распространить на краевую задачу (1.1)-(1.3) целый ряд положений качественной теории обыкновенных дифференциальных уравнений - теоремы Ляпунова и Андронова-Витта об устойчивости по первому приближению, теоремы об интегральных многообразиях и др.

1.2. В работе [1] явление диффузионной буферности для краевой задачи (1.1)-(1.3) установлено численными методами при значениях параметров

$$
h_{1}=0.8 ; \quad h_{2}=1.86 ; \quad r_{2}=0.79 ; \quad a=0.1 ; \quad 2.2 \leqslant r_{1} \leqslant 3 ; \quad 0.001 \leqslant D \leqslant 0.005
$$

(соответствуюших задаче лиса-мьшевидные). Цель настоящей работы - дать теоретическое объяснение этого явления в рамках задачи (1.1)-(1.3) при некоторых дополнительных ограничениях.

Для их формулировки выполним в (1.1)-(1.3) замены

$$
\frac{t}{h_{1}} \longrightarrow t, \quad N_{1}=1+u, \quad N_{2}=1+v,
$$

положим

$$
a=\varepsilon \ll 1, \quad \frac{r_{1} h_{1}}{1+a}=\frac{\pi}{2}+\gamma \varepsilon, \quad r_{2} h_{1}=r, \quad \frac{h_{2}}{h_{1}}=h, \quad h_{1} D \longrightarrow D,
$$

где знак параметра $\gamma \sim 1$ произволен, и будем считать, что

$$
r h<\frac{\pi}{2} .
$$

В результате вместо (1.1)-(1.3) получим краевую задачу

$$
\begin{gathered}
\frac{\partial u}{\partial t}=-\left(\frac{\pi}{2}+\gamma \varepsilon\right)[\varepsilon v+u(t-1, x)](1+u), \\
\frac{\partial v}{\partial t}=D \frac{\partial^{2} v}{\partial x^{2}}+r[u-v(t-h, x)](1+v), \\
\left.\frac{\partial v}{\partial x}\right|_{x=0}=\left.\frac{\partial v}{\partial x}\right|_{x=1}=0,
\end{gathered}
$$


являюшуюся объектом дальнейшего анализа. Отметим, что малость $a$ (см. (1.6)) наиболее естественна с биологической точки зрения [1], тогда как специальньй выбор $r_{1}$ в (1.6) и неравенство (1.7) обусловлены чисто математическими причинами, которые проясняются ниже.

Рассмотрим краевую задачу

$$
\frac{\partial u}{\partial t}=-\frac{\pi}{2} u(t-1, x), \quad \frac{\partial v}{\partial t}=D \frac{\partial^{2} v}{\partial x^{2}}+r[u-v(t-h, x)],\left.\quad \frac{\partial v}{\partial x}\right|_{\substack{x=0 \\ x=1}}=0,
$$

получающуюся из (1.8) при $\varepsilon=0$ после линеаризации на нулевом состоянии равновесия. Применяя к ней метод Фурье по системе функций $\cos n \pi x, n=0,1, \ldots$, убеждаемся, что спектр ее устойчивости на каждой моде $\cos n \pi x$ определяется из уравнений

$$
\lambda+\frac{\pi}{2} \exp (-\lambda)=0, \quad \lambda+r \exp (-\lambda h)=-n^{2} \pi^{2} D .
$$

Первое из них проанализировано, например, в [10], где показано, что все его корни (за исключением простой пары $\pm i \pi / 2$ ) лежат в полуплоскости $\operatorname{Re} \lambda<0$. Отрицательность же действительных частей корней второго уравнения (1.10) при любых $n$ и $D$ обеспечивает условие (1.7).

Итак, при $\varepsilon=0$ в спектре устойчивости нулевого состояния равновесия системы (1.8) появляется счетное число частот $\pm i \pi / 2$, а соответствуюшее им семейство 4-периодических решений линейной системы (1.9) имеет вид

$$
\begin{aligned}
& u=\xi(x) \exp \left(i \frac{\pi}{2} t\right)+\bar{\xi}(x) \exp \left(-i \frac{\pi}{2} t\right) \\
& v=\eta(x) \exp \left(i \frac{\pi}{2} t\right)+\bar{\eta}(x) \exp \left(-i \frac{\pi}{2} t\right), \quad \eta=-r L(\xi)
\end{aligned}
$$

где $\xi(x)$ - произвольная функция из $W_{2}^{1}[0,1]$,

$$
L=\left[D \frac{d^{2}}{d x^{2}}-\varkappa I\right]^{-1}, \quad \varkappa=\varkappa_{1}+i \varkappa_{2}, \quad \varkappa_{1}=r \cos \frac{\pi}{2} h, \quad \varkappa_{2}=\frac{\pi}{2}-r \sin \frac{\pi}{2} h
$$

$I$ - единичный оператор. Поэтому представляется естественным искать автоколебания краевой задачи (1.8) в виде рядов

$$
\begin{aligned}
u= & \sqrt{\varepsilon}\left[\xi(\tau, x) \exp \left(i \frac{\pi}{2} t\right)+\bar{\xi}(\tau, x) \exp \left(-i \frac{\pi}{2} t\right)\right] \\
& +\varepsilon u_{2}(t, \tau, x)+\varepsilon^{3 / 2} u_{3}(t, \tau, x)+\cdots, \\
v= & \sqrt{\varepsilon}\left[\eta(\tau, x) \exp \left(i \frac{\pi}{2} t\right)+\bar{\eta}(\tau, x) \exp \left(-i \frac{\pi}{2} t\right)\right]+\varepsilon v_{2}(t, \tau, x)+\cdots,
\end{aligned}
$$

где $\tau=\varepsilon t$ - медленное время, $\xi(\tau, x), \eta(\tau, x)=-r L(\xi)$-подлежащие определению амплитуды колебаний, а $u_{k}, v_{k}, k \geqslant 2,-$ тригонометрические полиномы от переменной $\pi t / 2$ с зависяшими от $\tau, x$ коэффициентами. 
Подставляя разложения (1.11) в задачу (1.8) и приравнивая коэффициенты при одинаковых степенях $\varepsilon$, последовательно находим

$$
\begin{gathered}
u_{2}=A(\tau, x) \exp (i \pi t)+\bar{A}(\tau, x) \exp (-i \pi t), \quad A=\frac{2-i}{5} \xi^{2}, \\
v_{2}=B(\tau, x) \exp (i \pi t)+\bar{B}(\tau, x) \exp (-i \pi t)+C(\tau, x), \\
B=-r\left[D \frac{d^{2}}{d x^{2}}-(i \pi+r \exp (-i \pi h)) I\right]^{-1}\left(A-\eta^{2} \exp \left(-i \frac{\pi}{2} h\right)\right), \\
C=2 r \cos \frac{\pi}{2} h\left[D \frac{d^{2}}{d x^{2}}-r I\right]^{-1}|\eta|^{2},
\end{gathered}
$$

а для $u_{3}$ получаем уравнение

$$
\begin{aligned}
\frac{\partial u_{3}}{\partial t}+\frac{\pi}{2} u_{3}(t-1, \tau, x)= & f_{1}(\tau, x) \exp \left(i \frac{\pi}{2} t\right)+\bar{f}_{1}(\tau, x) \exp \left(-i \frac{\pi}{2} t\right) \\
& +f_{2}(\tau, x) \exp \left(i \frac{3 \pi}{2} t\right)+\bar{f}_{2}(\tau, x) \exp \left(-i \frac{3 \pi}{2} t\right)
\end{aligned}
$$

Условие его разрешимости в нужном классе функций, т.е. равенство $f_{1}(\tau, x)=0$, приводит к интегрально-дифференциальной краевой задаче

$$
\begin{gathered}
\left(1+i \frac{\pi}{2}\right) \frac{\partial \xi}{\partial \tau}=\frac{\pi}{2} r L(\xi)+i \gamma \xi+\frac{\pi(1-3 i)}{10}|\xi|^{2} \xi \\
\left.\frac{\partial \xi}{\partial x}\right|_{x=0}=\left.\frac{\partial \xi}{\partial x}\right|_{x=1}=0
\end{gathered}
$$

для определения амплитуды $\xi(\tau, x)$. Всюду ниже фазовым пространством этой задачи считаем гильбертово пространство $W_{2}^{1}[0,1]$.

ТЕОРема 1.1. Предположим, что краевая задача (1.12) имеет автомодельный иикл

$$
\xi(\tau, x)=\xi_{0}(x) \exp \left(i \omega_{0} \tau\right)
$$

экспоненичально орбитально устойчивый или дихотомичный. Тогда при всех достаточно мальх $\varepsilon>0$ ему соответствует иикл краевой задачи (1.8) $с$ асимптотикой (1.11) и теми же свойствами устойчивости.

Справедливость теоремы достаточно просто устанавливается с помощью качественных соображений общего характера, имеюших место для краевой задачи (1.8) в силу сказанного выше. Действительно, разложим фазовое пространство $E$ в прямую сумму:

$$
E=V_{1} \oplus V_{2}, \quad V_{1}=P E, \quad V_{2}=(I-P) E,
$$


где проектор $P$ задается равенствами

$$
\begin{gathered}
P \varphi=\left(\xi(x) \exp \left(i \frac{\pi}{2} s\right)+\bar{\xi}(x) \exp \left(-i \frac{\pi}{2} s\right), \eta(x) \exp \left(i \frac{\pi}{2} s\right)+\bar{\eta}(x) \exp \left(-i \frac{\pi}{2} s\right)\right) \\
\xi(x)=\frac{1}{1+i \frac{\pi}{2}}\left(\varphi_{1}(0, x)+i \frac{\pi}{2} \int_{-1}^{0} \varphi_{1}(s, x) \exp \left(-i \frac{\pi}{2} s\right) d s\right) \\
\eta=-r L(\xi), \quad \varphi(s, x)=\left(\varphi_{1}(s, x), \varphi_{2}(s, x)\right) .
\end{gathered}
$$

Из этих формул следует, что $V_{1}, V_{2}$ - корневые подпространства системы (1.9) (инвариантные для ее траекторий), причем первое из них отвечает счетному числу чисто мнимых корней $\pm i \pi / 2$, а второе - всем остальным корням уравнений $(1.10)$. Таким образом, если $w_{1}=P \varphi, w_{2}=(I-P) \varphi$, то равенство $w_{2}=0$ задает бесконечномерное экспоненциально орбитально устойчивое инвариантное многообразие $\Sigma_{0}$ системы (1.9).

При переходе от линейной системы (1.9) к нелинейной краевой задаче (1.8) в некоторой достаточно малой окрестности нулевого состояния равновесия инвариантное многообразие $\Sigma_{0}$, естественно, сохраняется. Оно задается теперь равенством $w_{2}=G\left(w_{1}, \varepsilon\right)$, причем достаточно гладкое по совокупности переменных отображение $G(*, \varepsilon): V_{1} \rightarrow V_{2}$ таково, что

$$
G(0, \varepsilon) \equiv 0, \quad G_{w_{1}}^{\prime}(0,0)=0, \quad G_{\varepsilon}^{\prime}(0,0)=0
$$

(для доказательства можно воспользоваться схемой Хартмана [11], адаптированной для нелинейных полугрупп в банаховых пространствах в работе [12]). Отстается заметить, что в силу своего построения краевая задача (1.12) - укороченная нормальная форма на $\Sigma_{0}$ (отброшенный добавок является ограниченным нелинейным оператором в $W_{2}^{1}[0,1]$ порядка $\left.\varepsilon\right)$. Тем самым утверждение теоремы - непосредственное следствие предположенной грубости цикла (1.13).

В пространстве параметров

$$
\left\{(r, h, D, \gamma): r, h, D>0, \quad r h<\frac{\pi}{2}\right\}
$$

краевой задачи (1.12) введем в рассмотрение открытые множества $\Omega_{n}, n=1,2, \ldots$, определяюшиеся следующим образом. Будем говорить, что набор $(r, h, D, \gamma)$ принадлежит $\Omega_{n}$, если при этих значениях параметров краевая задача (1.12) имеет не менее $n$ пространственно неоднородных экспоненщиально орбитально устойчивых автомодельных циклов

$$
\xi_{k}(\tau, x)=\xi_{k}(x) \exp \left(i \omega_{k} \tau\right), \quad \xi_{k}(x) \not \equiv \text { const }, \quad k=1, \ldots, n
$$

Основной результат настоящей статьи - следующее утверждение. 
ТЕОРема 1.2 (о диффузионной буферности). Множество $\Omega_{n}$ не пусто при любом натуральном $n$.

Из теорем 1.1, 1.2 следует существование для каждого натурального $n$ и каждого набора параметров $(r, h, D, \gamma) \in \Omega_{n}$ такого числа $\varepsilon_{n}>0$, что при $0<\varepsilon \leqslant \varepsilon_{n}$ краевая задача (1.8) имеет $n$ экспоненциально орбитально устойчивых пространственно неоднородных циклов с асимптотикой (1.11), (1.14). Таким образом, проблема диффузионной буферности сводится к доказательству теоремы 1.2 , которому посвящен следуюший параграф.

В заключение добавим, что нулевая диффузия у $N_{1}$ (см. (1.1)) в нашем случае вполне естественна, так как подвижность мышевидных пренебрежимо мала. Учет же малой диффузии у жертвы не влияет на факт диффузионной буферности.

\section{§2. Доказательство теоремы 1.2}

2.1. Для удобства дальнейшего анализа в нормальной форме (1.12) последовательно вьполним замены

$$
\xi \exp (-i \gamma \tau) \longrightarrow \xi, \quad \frac{2 \pi r}{\pi^{2}+4} \tau \longrightarrow \tau, \quad \frac{\gamma\left(\pi^{2}+4\right)}{4 r} \longrightarrow \gamma, \quad \sqrt{\frac{\pi(3 \pi-2)}{5\left(\pi^{2}+4\right)}} \xi \longrightarrow \xi
$$

В результате получим краевую задачу

$$
\frac{\partial \xi}{\partial \tau}=\left(1-i \frac{\pi}{2}\right) L(\xi)+\gamma \xi-\left(1+i c_{0}\right)|\xi|^{2} \xi,\left.\quad \frac{\partial \xi}{\partial x}\right|_{\substack{x=0 \\ x=1}}=0
$$

где $c_{0}=(\pi+6) /(3 \pi-2) \approx 1.23122 \ldots$

Автоколебания краевой задачи (2.1) будем исследовать при дополнительном предположении о малости коэффициента диффузии $D$ (см. (1.2)). Точнее говоря, рассмотрим проблему существования и устойчивости пространственно неоднородных автомодельных циклов задачи (2.1), буфирцируюших из нулевого состояния равновесия при увеличении параметра $\gamma$. В связи с этим отметим, что нулевое решение нормальной формы (2.1) экспоненциально устойчиво при выполнении неравенств

$$
\varphi\left(D \pi^{2} n^{2}\right)<0, \quad n=0,1,2, \ldots,
$$

где

$$
\varphi(z)=\gamma-\operatorname{Re}\left[\frac{1-i \frac{\pi}{2}}{z+\varkappa}\right], \quad z \geqslant 0,
$$

и неустойчиво при выполнении хотя бы одного неравенства, строго противоположного (2.2).

Обратим внимание на следующее обстоятельство: если $\varphi\left(z_{0}\right)>0$ при некотором $z_{0} \geqslant 0$ (т.е. если функция (2.3) имеет участок положительности), то поставленная выше проблема нелокальна. В таком случае при $D \ll 1$ нулевое состояние равновесия задачи (2.1) неустойчиво сразу на асимптотически большом числе мод 

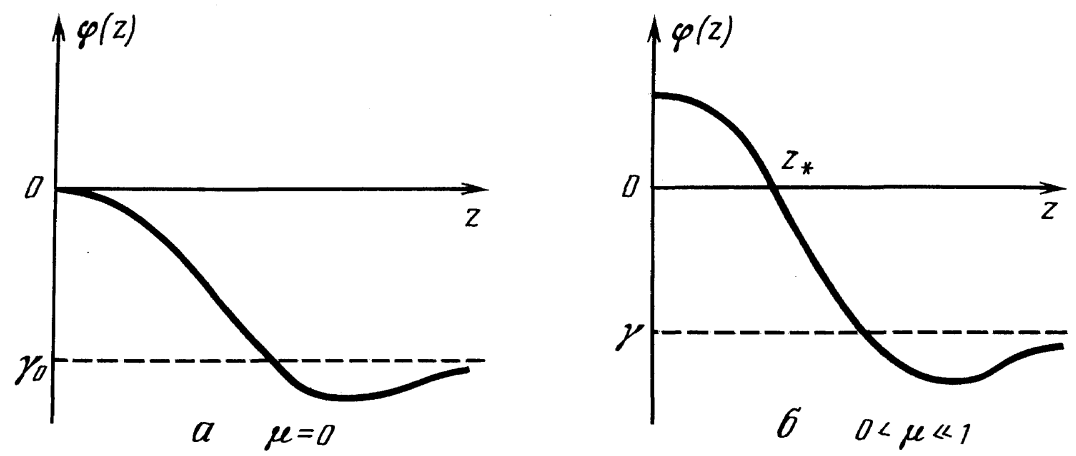

Рис. 1

$\cos n \pi x, n=0,1,2, \ldots$, причем соответствуюшие им характеристические показатели имеют порядок единица. Напротив, если функция (2.3) отрицательна при всех $z \geqslant 0$, то нулевое решение задачи (2.1) устойчиво при любом значении $D$. Таким образом, интересующая нас проблема становится локальной, если потребовать существование такого $z_{0} \geqslant 0$, что

$$
\begin{gathered}
\varphi\left(z_{0}\right)=\varphi^{\prime}\left(z_{0}\right)=0 ; \quad \varphi^{\prime \prime}\left(z_{0}\right)<0 ; \quad \varphi(z)<0 \text { при } z \neq z_{0} ; \\
\lim _{z \rightarrow \infty} \varphi(z)=\gamma<0 .
\end{gathered}
$$

Несложньй анализ приводит к выводу, что особенность $(2.4)$ в точке $z_{0}=0$ появляется при

$$
r=r_{0}, \quad \gamma=\gamma_{0},
$$

где

$$
\begin{gathered}
r_{0}=\frac{\frac{\pi}{2}\left(\sqrt{\frac{\pi^{2}}{4}+1}-\frac{\pi}{2}\right)}{-\cos \frac{\pi}{2} h+\left(\sqrt{\frac{\pi^{2}}{4}+1}-\frac{\pi}{2}\right) \sin \frac{\pi}{2} h}, \\
\gamma_{0}=\frac{\varkappa_{1}^{0}-\frac{\pi}{2} \varkappa_{2}^{0}}{\left(\varkappa_{1}^{0}\right)^{2}+\left(\varkappa_{2}^{0}\right)^{2}}, \quad \varkappa_{1}^{0}=r_{0} \cos \frac{\pi}{2} h, \quad \varkappa_{2}^{0}=\frac{\pi}{2}-r_{0} \sin \frac{\pi}{2} h,
\end{gathered}
$$

и при выполнении неравенств

$$
0<r_{0} h<\frac{\pi}{2}, \quad \varkappa_{2}^{0}>0
$$

Отметим еще, что в окрестности данной особенности мы находимся при значениях

$$
r=0.632, \quad h=2.325
$$

соответствующих набору параметров (1.5). Действительно, при $h=2.325$ получаем весьма близкое к $(2.7)$ значение $r_{0}=0.62667 \ldots$, удовлетворяющее неравенствам (2.6). 
Итак, положим в нормальной форме (2.1)

$$
D=\mu \ll 1, \quad r=r_{0}, \quad \gamma=\gamma_{0}+\delta \mu, \quad \delta>0,
$$

где параметр $\delta \sim 1$ характеризует "отход" от рассматриваемой особенности (отклонением порядка $\mu$ параметра $r$ от своего критического значения $r_{0}$ для простоты пренебрегаем, так как нас интересует динамика задачи (2.1) по $\gamma$ ). Подчеркнем, что при этом эволюция графика функции (2.3) имеет вид, показанный на рис.1: при $\mu>0$ появляется участок положительности $0 \leqslant z<z_{*}(\mu), z_{*}(\mu)=O(\sqrt{\mu})$. А отсюда вытекает строгое нарушение неравенств $(2.2)$ для асимптотически большого числа номеров $n=0,1, \ldots, n_{0}, n_{0} \sim \mu^{-1 / 4}$, т.е. моделируется отмеченная вьше нелокальная ситуация.

Последовательно выполняя при условиях (2.8) в задаче (2.1) замены

$$
\begin{gathered}
\xi \exp (-i \omega \tau) \longrightarrow \xi, \quad \frac{\xi}{\sqrt{\mu}} \longrightarrow \xi, \quad \mu \tau \longrightarrow \tau, \\
\omega=-\operatorname{Im}\left[\frac{1-i \frac{\pi}{2}}{\varkappa_{0}}\right], \quad \varkappa_{0}=\varkappa_{1}^{0}+i \varkappa_{2}^{0},
\end{gathered}
$$

получим краевую задачу

$$
\frac{\partial \xi}{\partial \tau}=\Pi(\mu) \xi+\delta \xi-\left(1+i c_{0}\right)|\xi|^{2} \xi,\left.\quad \frac{\partial \xi}{\partial x}\right|_{\substack{x=0 \\ x=1}}=0
$$

где

$$
\Pi(\mu)=-i \sigma_{0} \frac{d^{2}}{d x^{2}}\left[I-\frac{\mu}{\varkappa_{0}} \frac{d^{2}}{d x^{2}}\right]^{-1}, \quad \sigma_{0}=\operatorname{Im}\left[\frac{1-i \frac{\pi}{2}}{\varkappa_{0}^{2}}\right]>0
$$

(положительность $\sigma_{0}$ - следствие условий $(2.5),(2.6)$ ). Заметим, что краевая задача $(2.9)$ сингулярна, так как норма в смысле $W_{2}^{1}[0,1]$ ограниченного оператора $\Pi(\mu)$ имеет порядок $\mu^{-1}$.

Введем в рассмотрение краевую задачу

$$
\frac{\partial \xi}{\partial \tau}=-i \sigma_{0} \frac{\partial^{2} \xi}{\partial x^{2}}+\delta \xi-\left(1+i c_{0}\right)|\xi|^{2} \xi,\left.\quad \frac{\partial \xi}{\partial x}\right|_{\substack{x=0 \\ x=1}}=0
$$

предельную для задачи (2.9), так как для любой фиксированной функции $\xi(x) \in$ $W_{2}^{2}[0,1]$ в метрике $L_{2}(0,1)$ справедливо равенство

$$
\lim _{\mu \rightarrow 0} \Pi(\mu) \xi=-i \sigma_{0} \frac{d^{2} \xi}{d x^{2}}
$$

Ниже будем интересоваться существованием и устойчивостью автомодельных циклов предельной задачи (2.11) и их связью с аналогичными циклами исходной задачи $(2.9)$. 
2.2. Предположим, что краевая задача (2.11) имеет автомодельный цикл (1.13) с амплитудой $\xi_{0}(x) \not \equiv 0$. Вьполняя в уравнении $(2.11)$ замену

$$
\xi \exp \left(-i \omega_{0} \tau\right) \longrightarrow \xi
$$

переводящую этот цикл в состояние равновесия $\xi=\xi_{0}(x)$, и затем линеаризуя на нем это уравнение, получим спектральную задачу

$$
A w=\lambda w,\left.\quad \frac{d w}{d x}\right|_{x=0}=\left.\frac{d w}{d x}\right|_{x=1}=0,
$$

где

$$
\begin{gathered}
w=\operatorname{colon}\left(w^{1}, w^{2}\right), \\
A=\left(\begin{array}{cc}
-i \sigma_{0} & 0 \\
0 & i \sigma_{0}
\end{array}\right) \frac{d^{2}}{d x^{2}}+\left(\begin{array}{cc}
p(x) & q(x) \\
\bar{q}(x) & \bar{p}(x)
\end{array}\right), \\
p(x)=\delta-i \omega_{0}-2\left(1+i c_{0}\right)\left|\xi_{0}(x)\right|^{2}, \quad q(x)=-\left(1+i c_{0}\right) \xi_{0}^{2}(x) .
\end{gathered}
$$

Несложная проверка показьвает, что спектральная задача (2.13) всегда имеет нулевое собственное значение, которому отвечает собственная функция

$$
w_{0}(x)=\operatorname{colon}\left(i \xi_{0}(x),-i \bar{\xi}_{0}(x)\right)
$$

Ниже предполагается, что вьполнено следующее

УСловиЕ 1. Считаем, что $\lambda=0$ - простое собственное значение спектральной задачи (2.13).

Докажем теперь справедливость леммы, касающейся краевой задачи (2.9).

Лемма 2.1. Пусть выполнено условие 1. Тогда найдется такое число $\mu_{0}>0$, что при $0 \leqslant \mu \leqslant \mu_{0}$ краевая задача (2.9) имеет достаточно гладкий по совокупности переменных автомодельный цикл

$$
\xi(\tau, x, \mu)=\xi(x, \mu) \exp (i \omega(\mu) \tau)
$$

обращающийся при $\mu=0$ в цикл (1.13) предельной задачи (2.11).

ДоКАЗАТЕЛЬСТво. Вьполняя в уравнении (2.9) замену, аналогичную (2.12), для определения амплитуды $\xi(x, \mu)$ и частоты $\omega(\mu)$ интересуюшего нас цикла получим краевую задачу

$$
\Pi(\mu) \xi+(\delta-i \omega) \xi-\left(1+i c_{0}\right)|\xi|^{2} \xi=0,\left.\quad \frac{d \xi}{d x}\right|_{\substack{x=0 \\ x=1}}=0 .
$$

Применив к обеим частям уравнения (2.17) оператор

$$
B=I-\frac{\mu}{\varkappa_{0}} \frac{d^{2}}{d x^{2}}
$$


придем к эквивалентной краевой задаче

$$
\begin{aligned}
-i \sigma_{0} \frac{d^{2} \xi}{d x^{2}}+ & (\delta-i \omega) \xi-\left(1+i c_{0}\right)|\xi|^{2} \xi \\
& =\frac{\mu}{\varkappa_{0}}\left[-i \omega \xi+\delta \frac{d^{2} \xi}{d x^{2}}-\left(1+i c_{0}\right) \frac{d^{2}}{d x^{2}}\left(|\xi|^{2} \xi\right)\right],\left.\quad \frac{d \xi}{d x}\right|_{\substack{x=0 \\
x=1}}=0
\end{aligned}
$$

Обратим внимание на то, что, в отличие от задачи (2.17), зависимость от $\mu$ краевой задачи (2.19) регулярна, так как стоящий в правой части уравнения (2.19) оператор эквивалентен оператору $d^{2} / d x^{2}$. Тем самым для доказательства существования решения

$$
(\xi(x, \mu), \omega(\mu)): \quad \xi(x, 0)=\xi_{0}(x), \quad \omega(0)=\omega_{0}
$$

задачи (2.19) можно воспользоваться стандартной схемой, основные этапы которой описываются ниже.

Дополним краевую задачу (2.19) аналогичной краевой задачей для $\bar{\xi}$ и положим в получившейся системе уравнений

$$
\xi=\xi_{0}(x)+w^{1}, \quad \bar{\xi}=\bar{\xi}_{0}(x)+w^{2}, \quad \omega=\omega_{0}+\psi, \quad w=\operatorname{colon}\left(w^{1}, w^{2}\right) .
$$

В итоге приходим к краевой задаче (ср. с (2.13))

$$
A w=\Phi(w, \psi, \mu),\left.\quad \frac{d w}{d x}\right|_{x=0}=\left.\frac{d w}{d x}\right|_{x=1}=0
$$

где

$$
\begin{gathered}
\Phi=\operatorname{colon}\left(\Phi^{1}, \Phi^{2}\right), \\
\Phi^{1}=i \psi\left(\xi_{0}+w^{1}\right)+\left(1+i c_{0}\right)\left(\bar{\xi}_{0}\left(w^{1}\right)^{2}+2 \xi_{0} w^{1} w^{2}+\left(w^{1}\right)^{2} w^{2}\right) \\
+\frac{\mu}{\varkappa_{0}}\left[-i\left(\omega_{0}+\psi\right)\left(\xi_{0}+w^{1}\right)+\delta \frac{d^{2}}{d x^{2}}\left(\xi_{0}+w^{1}\right)\right. \\
\left.-\left(1+i c_{0}\right) \frac{d^{2}}{d x^{2}}\left(\left(\xi_{0}+w^{1}\right)^{2}\left(\bar{\xi}_{0}+w^{2}\right)\right)\right] \\
\Phi^{2}=-i \psi\left(\bar{\xi}_{0}+w^{2}\right)+\left(1-i c_{0}\right)\left(\xi_{0}\left(w^{2}\right)^{2}+2 \bar{\xi}_{0} w^{1} w^{2}+\left(w^{2}\right)^{2} w^{1}\right) \\
+\frac{\mu}{\bar{\varkappa}_{0}}\left[i\left(\omega_{0}+\psi\right)\left(\bar{\xi}_{0}+w^{2}\right)+\delta \frac{d^{2}}{d x^{2}}\left(\bar{\xi}_{0}+w^{2}\right)\right. \\
\left.-\left(1-i c_{0}\right) \frac{d^{2}}{d x^{2}}\left(\left(\bar{\xi}_{0}+w^{2}\right)^{2}\left(\xi_{0}+w^{1}\right)\right)\right] .
\end{gathered}
$$

Рассмотрим вопрос об обратимости оператора $A$ (см. (2.14)). С этой целью обозначим через $g_{0}(x)$ собственную функцию сопряженного оператора $A^{*}$, отвечающую нулевому собственному значению и удовлетворяющую условию нормировки

$$
\int_{0}^{1}\left(w_{0}(x), g_{0}(x)\right) d x=1
$$


здесь $(*, *)$ - обычное скалярное произведение в $\mathbb{C}^{2}$, а $w_{0}(x)$ - функция $(2.15)$. Отметим, что возможность такой нормировки - следствие предполагаемой в условии 1 простоты нулевого собственного значения.

Из условия 1 также вытекает, что неоднородная краевая задача

$$
A w=f(x),\left.\quad \frac{d w}{d x}\right|_{\substack{x=0 \\ x=1}}=0, \quad f(x) \in L_{2}\left([0,1] ; \mathbb{C}^{2}\right)
$$

разрешима тогда и только тогда, когда функция $f(x)$ ортогональна в среднем функции $g_{0}(x)$. При этом решение $w_{f}(x)$ указанной неоднородной краевой задачи, ортогональное в среднем функции $g_{0}(x)$, единственно и удовлетворяет неравенству коэриитивности [13]

$$
\left\|w_{f}\right\|_{W_{2}^{2}\left([0,1] ; \mathbb{C}^{2}\right)} \leqslant M\|f\|_{L_{2}\left([0,1] ; \mathbb{C}^{2}\right)},
$$

где постоянная $M>0$ не зависит от выбора $f$.

Обозначим через $E_{0}$ подпространство гильбертова пространства $W_{2}^{2}\left([0,1] ; \mathbb{C}^{2}\right)$, состоящее из функций $w(x)$, ортогональных в среднем функции $g_{0}(x)$ и удовлетворяющих граничным условиям $w_{x}^{\prime}=0$ при $x=0$ и при $x=1$. Из сказанного выше следует, что оператор $A$ имеет ограниченный обратный, действующий из $L_{2}^{0}\left([0,1] ; \mathbb{C}^{2}\right)$ в $E_{0}$ (здесь $L_{2}^{0} \subset L_{2}\left([0,1] ; \mathbb{C}^{2}\right)$ - подпространство функций, ортогональных в среднем функции $\left.g_{0}(x)\right)$. Поэтому в предположении, что $w \in E_{0}$, краевая задача (2.20) эквивалентна системе уравнений

$$
w=\Psi_{1}(w, \psi, \mu), \quad \Psi_{2}(w, \psi, \mu)=0,
$$

где $(w, \psi, \mu) \in E_{0} \times \mathbb{C} \times \mathbb{R}$,

$$
\Psi_{1}=A^{-1}\left[\Phi-\int_{0}^{1}\left(\Phi, g_{0}(x)\right) d x \cdot w_{0}(x)\right], \quad \Psi_{2}=\int_{0}^{1}\left(\Phi, g_{0}(x)\right) d x .
$$

Из структуры выражений (2.21) и неравенства коэрцитивности видно, что операторы

$$
\Psi_{1}: E_{0} \times \mathbb{C} \times \mathbb{R} \longrightarrow E_{0}, \quad \Psi_{2}: E_{0} \times \mathbb{C} \times \mathbb{R} \longrightarrow \mathbb{C}
$$

являются ограниченными и гладкими (по Фреше) по всем своим аргументам. Формулы (2.21) также показывают, что к системе $(2.22)$ в точке $(0,0,0)$ применима теорема о неявной функции, доставляюшая ее решение

$$
\begin{gathered}
w(x, \mu)=\operatorname{colon}\left(w^{1}(x, \mu), w^{2}(x, \mu)\right), \quad \psi=\psi(\mu), \\
w^{1}(x, 0) \equiv w^{2}(x, 0) \equiv 0, \quad \psi(0)=0 .
\end{gathered}
$$

Наконец, отметим, что наряду с этим решением системе (2.22) будет удовлетворять и набор фуннций

$$
w(x, \mu)=\operatorname{colon}\left(\bar{w}^{2}(x, \mu), \bar{w}^{1}(x, \mu)\right), \quad \psi=\bar{\psi}(\mu)
$$

(для доказательства достаточно перейти в $(2.20),(2.21)$ к комплексному сопряжению). А так как решение вида (2.23) единственно, то $\bar{w}^{2}(x, \mu) \equiv w^{1}(x, \mu)$, $\psi(\mu) \equiv \bar{\psi}(\mu)$. Лемма доказана. 
2.3. Займемся теперь анализом свойств устойчивости цикла (2.16). Для этого потребуется одно вспомогательное утверждение.

ЛЕмма 2.2. Найдется такое достаточно большое натуральное число $n_{0}$, что каждому $n \geqslant n_{0}$ соответствует пара собственных значений $\lambda_{n}, \bar{\lambda}_{n}$ краевой задачи (2.13) с асимптотикой

$$
\lambda_{n}=i \sigma_{0} \pi^{2} n^{2}+p_{0}+O\left(\frac{1}{n}\right), \quad p_{0}=\int_{0}^{1} p(x) d x, \quad n \longrightarrow \infty
$$

При әтом асимптотика (в метрике $\left.L_{2}\left([0,1] ; \mathbb{C}^{2}\right)\right)$ отвечающих им нормированных единицей собственных функиий имеет вид

$e_{n}^{1}(x)=\left(\begin{array}{l}1 \\ 0\end{array}\right) \sqrt{2} \cos \pi n x+O\left(\frac{1}{n}\right), \quad e_{n}^{2}(x)=\left(\begin{array}{l}0 \\ 1\end{array}\right) \sqrt{2} \cos \pi n x+O\left(\frac{1}{n}\right), \quad n \rightarrow \infty$.

ДоказАтельство. Результаты монографии [14] показывают, что главные члены асимптотики собственных значений и собственных функций оператора $A$ (см. (2.14)) определяются по его главной части $\operatorname{diag}\left\{-i \sigma_{0} d^{2} / d x^{2}, i \sigma_{0} d^{2} / d x^{2}\right\}$. Поэтому положим в (2.13)

$$
w=w_{n}(x)+y, \quad \lambda=i \sigma_{0} \pi^{2} n^{2}+p_{0}+\psi, \quad w_{n}(x)=\left(\begin{array}{l}
1 \\
0
\end{array}\right) \sqrt{2} \cos \pi n x,
$$

где $y$ и $\psi$ - поправки к собственной функции и собственному значению. В результате приходим к краевой задаче

$$
A_{0} y+A_{1} y+A_{1} w_{n}(x)=\psi y+\psi w_{n}(x),\left.\quad \frac{d y}{d x}\right|_{\substack{x=0 \\ x=1}}=0
$$

где

$$
\begin{gathered}
A_{0}=\operatorname{diag}\left\{-i \sigma_{0} \frac{d^{2}}{d x^{2}}-i \sigma_{0} \pi^{2} n^{2} I, i \sigma_{0} \frac{d^{2}}{d x^{2}}-\left(i \sigma_{0} \pi^{2} n^{2}+2 i \operatorname{Im} p_{0}\right) I\right\}, \\
A_{1}=\left(\begin{array}{cc}
p(x)-p_{0} & q(x) \\
\bar{q}(x) & \bar{p}(x)-\bar{p}_{0}
\end{array}\right) .
\end{gathered}
$$

Из диагональной структуры оператора $A_{0}$ следует как его обратимость в подпространстве $L_{2}^{0} \subset L_{2}\left([0,1] ; \mathbb{C}^{2}\right)$ функций, ортогональных в среднем $w_{n}(x)$, так и оценка

$$
\left\|A_{0}^{-1}\right\|_{L_{2}^{0} \rightarrow L_{2}^{0}} \leqslant \frac{M}{n} .
$$

(Здесь и ниже одной и той же буквой $M$ обозначаются различные не зависящие от $n$ положительные постоянные, точные значения которых несущественны.) 
Перейдем, далее, от уравнения $(2.26)$ к вспомогательному уравнению в $L_{2}^{0}$ :

$$
\begin{gathered}
y=A_{0}^{-1}\left\{\Psi-\int_{0}^{1}\left(\Psi, w_{n}(x)\right) d x \cdot w_{n}(x)\right\}, \\
\Psi=\psi y-A_{1} y-A_{1} w_{n}(x)+\psi w_{n}(x) .
\end{gathered}
$$

Оценка (2.27) позволяет применить к нему приншип сжимающих отображений и получить поправку к собственной функции $w$ - функцию $y(x, \psi)$, удовлетворяющую неравенствам

$$
\|y\|_{L_{2}^{0}} \leqslant \frac{M}{n}, \quad\left\|\frac{\partial y}{\partial \psi}\right\|_{L_{2}^{0}} \leqslant \frac{M}{n} .
$$

Наконец, займемся нахождением поправки $\psi$ к собственному значению $\lambda$ из уравнения

$$
\int_{0}^{1}\left(\Psi, w_{n}(x)\right) d x=0 .
$$

Подставляя в него функцию $y(x, \psi)$ и учитывая оценки $(2.28)$ вместе с равенством

$$
\int_{0}^{1}\left(A_{1} w_{n}(x), w_{n}(x)\right) d x=\int_{0}^{1} p(x) \cos 2 \pi n x d x,
$$

убеждаемся, что это уравнение однозначно разрешимо и его решение имеет порядок $\psi=O(1 / n), n \rightarrow \infty$. Лемма доказана.

Введем теперь еше одно нужное для дальнейшего

УСловиЕ 2. Предполагаем, что все ненулевые собственные значения краевой задачи (2.13) имеют отрицательные действительные части и вьполняется неравенство (см. (2.24))

$$
\operatorname{Re} p_{0}<0 .
$$

Смысл условия 2 проясняет лемма 2.2. Действительно, пусть $\Lambda$ - совокупность всех ненулевых собственных значений краевой задачи (2.13). Из формул (2.24) следует, что условие 2 эквивалентно неравенству

$$
\Delta_{0}=-\sup _{\lambda \in \Lambda} \operatorname{Re} \lambda>0 .
$$

Введем в рассмотрение аналогичную (2.13) краевую задачу

здесь

$$
A(\mu) w=\lambda w,\left.\quad \frac{d w}{d x}\right|_{x=0}=\left.\frac{d w}{d x}\right|_{x=1}=0
$$

где (ср. с (2.10), (2.18))

$$
A(\mu)=\left(\begin{array}{cc}
\Pi(\mu) & 0 \\
0 & \bar{\Pi}(\mu)
\end{array}\right)+\left(\begin{array}{cc}
p(x, \mu) & q(x, \mu) \\
\bar{q}(x, \mu) & \bar{p}(x, \mu)
\end{array}\right),
$$

$$
\bar{\Pi}(\mu)=i \sigma_{0} \frac{d^{2}}{d x^{2}} \bar{B}^{-1}, \quad \bar{B}=I-\frac{\mu}{\bar{\varkappa}_{0}} \frac{d^{2}}{d x^{2}},
$$

а функции $p(x, \mu), q(x, \mu)$ определяются по формулам, аналогичным $(2.14)$ с заменой $\xi_{0}(x), \omega_{0}$ на $\xi(x, \mu), \omega(\mu)$. Отметим, что, как и (2.13), краевая задача $(2.29)$ имеет нулевое собственное значение, которому отвечает собственная функция

$$
w_{0}(x, \mu)=\operatorname{colon}(i \xi(x, \mu),-i \bar{\xi}(x, \mu)) .
$$


ЛЕмма 2.3. Пусть выполнены условия 1 и 2. Тогда по любому фиксированному числу $\Delta \in\left(0, \Delta_{0}\right)$ можно указать такое число $\mu_{0}=\mu_{0}(\Delta)>0$, что при $0 \leqslant \mu \leqslant \mu_{0}$ все собственные значения (кроме простого нулевого) краевой задачи (2.29) лежат в полуплоскости $\operatorname{Re} \lambda \leqslant-\Delta$.

ДоКАЗАТЕЛЬСТво леммы базируется на тех же идеях, что и обоснование лемм 2.1 и 2.2.

Сначала разберемся с аналогами собственных значений (2.24). Заметим, что главные члены асимптотики (2.25) являются собственными функциями главной части $\operatorname{diag}\{\Pi(\mu), \bar{\Pi}(\mu)\}$ оператора (2.30). Поэтому найдется такое достаточно большое (не зависящее от $\mu$ ) натуральное $n_{0}$, что каждому $n \geqslant n_{0}$ соответствует пара $\lambda_{n}(\mu), \bar{\lambda}_{n}(\mu), n \geqslant n_{0}$, собственных значений краевой задачи $(2.29)$ с асимптотикой при $n \rightarrow \infty$ :

$$
\operatorname{Re} \lambda_{n}(\mu)= \begin{cases}\frac{1}{\mu} \varphi\left(\mu \pi^{2} n^{2}\right)+p_{0}+O\left(\frac{1}{n}+\mu\right), & n_{0} \leqslant n \leqslant \frac{c}{\sqrt{\mu}}, \\ \frac{1}{\mu} \varphi\left(\mu \pi^{2} n^{2}\right)+O(1), & n \geqslant \frac{c}{\sqrt{\mu}} .\end{cases}
$$

Здесь $\varphi(z)$ - функция (2.3) при условиях $(2.5) ; c>0$ - произвольная фиксированная постоянная; $p_{0}-$ величина, введенная в (2.24). Доказательство существования собственных значений $\lambda_{n}(\mu), \bar{\lambda}_{n}(\mu), n \geqslant n_{0}$, с асимптотикой (2.31) проводится по той же схеме, что и обоснование формул $(2.24),(2.25)$.

Учитьвая в асимптотических формулах (2.31) свойства функции $\varphi(z)$ (см. рис. 1a) и неравенство из условия 2 , убеждаемся в существовании такого числа $\mu_{0}>0$, что при $0 \leqslant \mu \leqslant \mu_{0}$ все собственные значения $\lambda_{n}(\mu), \bar{\lambda}_{n}(\mu), n \geqslant n_{0}$, лежат в полуплоскости $\operatorname{Re} \lambda \leqslant-\Delta$.

Обозначим через $\lambda_{k}(\mu), k=1, \ldots, k_{0}$, отличные от $\lambda_{n}(\mu), \bar{\lambda}_{n}(\mu), n \geqslant n_{0}$, собственные значения краевой задачи $(2.29)$ (номер $k_{0}$ не зависит от $\mu$ ). Для того чтобы разобраться с их расположением, применим к уравнению (2.29) оператор $\operatorname{diag}\{B, \bar{B}\}$ (см. (2.18)). В результате этого сингулярно возмущенная краевая задача (2.29) переходит в регулярно зависящую от $\mu$ спектральную задачу

$$
A w-\lambda w=\mu H_{1}(\mu) w-\mu \lambda H_{2} w,\left.\quad \frac{d w}{d x}\right|_{x=0}=\left.\frac{d w}{d x}\right|_{x=1}=0,
$$

где

$$
\begin{aligned}
H_{1}(\mu)= & \frac{1}{\mu}\left(\begin{array}{cc}
p(x)-p(x, \mu) & q(x)-q(x, \mu) \\
\bar{q}(x)-\bar{q}(x, \mu) & \bar{p}(x)-\bar{p}(x, \mu)
\end{array}\right) \\
& +\left(\begin{array}{cc}
\frac{1}{\varkappa_{0}} \frac{d^{2}}{d x^{2}} & 0 \\
0 & \frac{1}{\varkappa_{0}} \frac{d^{2}}{d x^{2}}
\end{array}\right)\left(\begin{array}{cc}
p(x, \mu) & q(x, \mu) \\
\bar{q}(x, \mu) & \bar{p}(x, \mu)
\end{array}\right), \\
& H_{2}=\operatorname{diag}\left\{\frac{1}{\varkappa_{0}} \frac{d^{2}}{d x^{2}}, \frac{1}{\bar{\varkappa}_{0}} \frac{d^{2}}{d x^{2}}\right\} .
\end{aligned}
$$

Из теории возмущений [15], справедливой для краевой задачи (2.32) в силу ее регулярности, заключаем, что, во-первых, при всех достаточно малых $\mu$ ее нулевое собственное значение является простым (что следует из условия 1), а во-вторых, остальные собственные значения $\lambda_{k}(\mu), k=1, \ldots, k_{0}$, при $\mu \rightarrow 0$ стремятся к соответствуюшим собственным значениям предельной задачи (2.13). Лемма доказана. 
2.4. Леммы 2.1 и 2.3 сводят проблему диффузионной буферности к вопросу о сушествовании и устойчивости автомодельных циклов у предельной краевой задачи (2.11). Решение этого вопроса, представляюшего и самостоятельный интерес, дается ниже.

Выполняя в уравнении (2.11) подстановку $\xi=\xi(x) \exp (i \omega \tau)$, для нахождения пар $(\xi(x), \omega)$ получаем краевую задачу

$$
i \sigma_{0} \frac{d^{2} \xi}{d x^{2}}+i \omega \xi=\delta \xi-\left(1+i c_{0}\right)|\xi|^{2} \xi,\left.\quad \frac{d \xi}{d x}\right|_{\substack{x=0 \\ x=1}}=0,
$$

исследование которой проведем с помошью так называемого принципа подобия .

Зафиксируем произвольное натуральное $n$ и положим в (2.33)

$$
y=n x, \quad \omega=\sigma_{0} \pi^{2} n^{2}+\omega_{0} .
$$

В результате приходим к краевой задаче

$$
\begin{aligned}
i \sigma_{0}\left(\frac{d^{2} \xi}{d y^{2}}+\pi^{2} \xi\right) & =\alpha\left[\left(\delta-i \omega_{0}\right) \xi-\left(1+i c_{0}\right)|\xi|^{2} \xi\right] \\
\left.\frac{d \xi}{d y}\right|_{y=0} & =\left.\frac{d \xi}{d y}\right|_{y=n}=0
\end{aligned}
$$

где $\alpha=1 / n^{2}$. Перейдем, далее, от (2.35) к вспомогательной краевой задаче

$$
\begin{aligned}
i \sigma_{0}\left(\frac{d^{2} \xi}{d y^{2}}+\pi^{2} \xi\right) & =\alpha\left[\left(\delta-i \omega_{0}\right) \xi-\left(1+i c_{0}\right)|\xi|^{2} \xi\right] \\
\left.\frac{d \xi}{d y}\right|_{y=0} & =\left.\frac{d \xi}{d y}\right|_{y=1}=0 .
\end{aligned}
$$

Принцип подобия состоит в следуюшем: если $\left(\xi_{0}(y), \omega_{0}\right)$ - решение краевой задачи $(2.36)$, то после продолжения функции $\xi_{0}(y)$ на отрезок $[-1,0]$ по четности и затем на всю ось по периодичности с периодом 2 пара $\left(\xi_{0}(y), \omega_{0}\right)$ становится решением краевой задачи (2.35). Тем самым анализу подлежит вспомогательная краевая задача (2.36), в которой для удобства будем считать параметр $\alpha$ меняюшимся непрерывно.

В формулируемом ниже утверждении предполагаем, что

$$
0<\alpha \ll 1 \text {. }
$$

Лемма 2.4. Найдется такое число $\alpha_{0}>0$, что при всех $0 \leqslant \alpha \leqslant \alpha_{0}$ существует единственная пара достаточно гладких по своим аргументам функиий

$$
\left(\xi(y, \alpha), \omega_{0}(\alpha)\right), \quad \xi(y, 0)=\sqrt{\frac{4 \delta}{3}} \cos \pi y, \quad \omega_{0}(0)=-\delta c_{0},
$$

удовлетворяющая краевой задаче (2.36). 
ДоКАЗАТЕЛЬСТВО. Изложим сначала асимптотическую часть обоснования леммы. Подставляя в соотношения (2.36) ряды

$$
\xi(y, \alpha)=\xi_{0} \cos \pi y+\alpha \xi_{1}(y)+\cdots, \quad \omega_{0}(\alpha)=\omega_{0,0}+\alpha \omega_{0,1}+\cdots
$$

где $\xi_{0}, \omega_{0,0}$ - подлежащие определению вещественные постоянные, и приравнивая коэффициенты при $\alpha$, получим краевую задачу

$$
\begin{aligned}
i \sigma_{0}\left(\frac{d^{2} \xi_{1}}{d y^{2}}+\pi^{2} \xi\right) & =\left(\delta-i \omega_{0,0}\right) \xi_{0} \cos \pi y-\left(1+i c_{0}\right) \xi_{0}^{3} \cos ^{3} \pi y \\
\left.\frac{d \xi_{1}}{d y}\right|_{y=0} & =\left.\frac{d \xi_{1}}{d y}\right|_{y=1}=0 .
\end{aligned}
$$

Условие ее разрешимости, имеюшее вид

$$
\left(\delta-i \omega_{0,0}\right) \xi_{0}-\frac{3}{4}\left(1+i c_{0}\right) \xi_{0}^{3}=0
$$

приводит к равенствам, определяющим нужные константы:

$$
\xi_{0}=\sqrt{\frac{4 \delta}{3}}, \quad \omega_{0,0}=-\delta c_{0}
$$

Учитывая их в правой части уравнения (2.39), находим

$$
\xi_{1}(y)=-\frac{i\left(1+i c_{0}\right) \xi_{0}^{3}}{32 \pi^{2} \sigma_{0}} \cos 3 \pi y+\xi_{1} \cos \pi y
$$

где неизвестная вешественная постоянная $\xi_{1}$ вместе с поправкой к частоте $\omega_{0,1}$ определяются из условия разрешимости аналогичной (2.39) краевой задачи для $\xi_{2}(y)$ и т.д.

Доказательство существования и единственности решения (2.37) с асиптотикой (2.38) сводится к теореме о неявной функции с помошью метода, описанного при обосновании леммы 2.1. Подчеркнем, что в данном случае он применяется в более простой ситуации, когда порождающее уравнение (получающееся из (2.35) при $\alpha=0)$ линейно. Лемма доказана.

Эта лемма вместе с принципом подобия обеспечивает существование у краевой задачи (2.11) счетного числа быстро осциллирующих автомодельных циклов

$$
\xi_{n}(\tau, x)=\xi_{n}(x) \exp \left(i \omega_{n} \tau\right), \quad n \geqslant \frac{1}{\sqrt{\alpha_{0}}}
$$

где

$$
\xi_{n}(x)=\left.\xi(y, \alpha)\right|_{y=n x, \alpha=1 / n^{2}}, \quad \omega_{n}=\sigma_{0} \pi^{2} n^{2}+\left.\omega_{0}(\alpha)\right|_{\alpha=1 / n^{2}} .
$$


2.5. Для исследования устойчивости автомодельного цикла (2.40) с произвольно фиксированным достаточно большим номером $n$ рассмотрим отвечающую ему краевую задачу (2.13). Выполним в ней замены (2.34), $\lambda / n^{2} \rightarrow \lambda$ и будем считать, как и ранее, параметр $\alpha=1 / n^{2}$ непрерывно меняющимся и достаточно малым. После отбрасывания членов порядка малости $\alpha^{2}$ и выше получим краевую задачу

$$
\Lambda_{0}\left(\frac{d^{2} w}{d y^{2}}+\pi^{2} w\right)+\alpha \Lambda_{1}(y) w=\lambda w,\left.\quad \frac{d w}{d y}\right|_{y=0}=\left.\frac{d w}{d y}\right|_{y=n}=0
$$

где

$$
\begin{gathered}
w=\operatorname{colon}\left(w^{1}, w^{2}\right) \\
\Lambda_{0}=\operatorname{diag}\left\{-i \sigma_{0}, i \sigma_{0}\right\}, \quad \Lambda_{1}=\left(\begin{array}{cc}
p(y) & q(y) \\
\bar{q}(y) & \bar{p}(y)
\end{array}\right), \\
p(y)=-\frac{\delta}{3}\left(1+i c_{0}\right)(1+4 \cos 2 \pi y), \quad q(y)=-\frac{2 \delta}{3}\left(1+i c_{0}\right)(1+\cos 2 \pi y) .
\end{gathered}
$$

При $\alpha=0$ спектр краевой задачи (2.41) ясен: это цепочка чисто мнимых собственных значений

$$
\lambda_{0}(\beta), \quad \bar{\lambda}_{0}(\beta) ; \quad \lambda_{0}(\beta)=i \sigma_{0} \pi^{2}\left(\beta^{2}-1\right), \quad \beta=\frac{k}{n}, \quad k=0,1,2, \ldots,
$$

которым отвечают собственные функции

$$
\operatorname{colon}(\cos \pi \beta y, 0), \quad \operatorname{colon}(0, \cos \pi \beta y) .
$$

Таким образом, проблема устойчивости циклов (2.40) сводится к исследованию асимптотики собственных значений $(2.42)$ при $\alpha \ll 1$, равномерной по параметру $\beta \geqslant 0$.

Основная трудность, возникающая на этом пути, связана с сингулярностью краевой задачи (2.41), так как она рассматривается на асимптотически большом отрезке $0 \leqslant y \leqslant n$. Для преодоления указанной трудности используем следующий специальный прием.

Положим в соотношениях (2.41)

$$
w=\eta_{1}(y) \cos \pi \beta y-\eta_{2}(y) \sin \pi \beta y,
$$

где $\eta_{j}(y), j=1,2,-$ вектор-функции со значениями в $\mathbb{C}^{2}$. В результате для $\eta=$ $\operatorname{colon}\left(\eta_{1}, \eta_{2}\right)$ получается регулярная краевая задача

$$
\begin{gathered}
B_{0}\left(\frac{d^{2} \eta}{d y^{2}}+\pi^{2}\left(1-\beta^{2}\right) \eta\right)+2 \pi \beta B_{1} \frac{d \eta}{d y}+\alpha B_{2}(y) \eta=\lambda \eta \\
\left.\frac{d \eta_{1}}{d y}\right|_{y=0}=\left.\frac{d \eta_{1}}{d y}\right|_{y=1}=\left.\eta_{2}\right|_{y=0}=\left.\eta_{2}\right|_{y=1}=0
\end{gathered}
$$


где четырехмерные матрицы $B_{j}, j=0,1,2$, задаются равенствами

$$
B_{0}=\operatorname{diag}\left\{\Lambda_{0}, \Lambda_{0}\right\}, \quad B_{1}=\left(\begin{array}{cc}
0 & -\Lambda_{0} \\
\Lambda_{0} & 0
\end{array}\right), \quad B_{2}=\operatorname{diag}\left\{\Lambda_{1}, \Lambda_{1}\right\}
$$

параметр $\beta \geqslant 0$ в силу произвольности чисел $k, n$ будем считать меняющимся непрерьвно.

Остановимся на связи краевых задач (2.41) и (2.44). Предположим, что на некотором промежутке $J$ изменения параметра $\beta$ вспомогательная краевая задача (2.44) имеет собственное значение $\lambda=\lambda(\beta)$, которому отвечает собственная функция

$$
\eta(y, \beta)=\operatorname{colon}\left(\eta_{1}(y, \beta), \eta_{2}(y, \beta)\right)
$$

Тогда, продолжая ее компоненты $\eta_{j}(y, \beta), j=1,2$, на отрезок $-1 \leqslant y \leqslant 0$ по четности и нечетности соответственно, а затем на всю ось по периодичности с периодом 2, убеждаемся, что построенная по (2.45) функция (2.43) при

$$
\beta=\frac{k}{n} \in J, \quad \lambda=\left.\lambda(\beta)\right|_{\beta=k / n}
$$

удовлетворяет исходной краевой задаче (2.41).

Заметим, далее, что при $\alpha=0$ краевая задача (2.44) также имеет собственные значения (2.42), которым отвечают собственные функции

$$
\operatorname{colon}(1,0,0,0), \quad \operatorname{colon}(0,1,0,0) .
$$

Поэтому обсуждаемая проблема устойчивости сводится к построению асимптотики этих собственных значений уже в рамках регулярной краевой задачи (2.44).

2.6. При построении асимптотики собственных значений

$$
\lambda(a, \beta), \quad \lambda(0, \beta)=\lambda_{0}(\beta)
$$

краевой задачи (2.44) естественно возникают два различных случая: в зависимости от того, простым или кратным является собственное значение $\lambda_{0}(\beta)($ см. $(2.42))$ предельной краевой задачи

$$
\begin{gathered}
B_{0}\left(\frac{d^{2} \eta}{d y^{2}}+\pi^{2}\left(1-\beta^{2}\right) \eta\right)+2 \pi \beta B_{1} \frac{d \eta}{d y}-\lambda \eta=0 \\
\left.\frac{d \eta_{1}}{d y}\right|_{y=0}=\left.\frac{d \eta_{1}}{d y}\right|_{y=1}=\left.\eta_{2}\right|_{y=0}=\left.\eta_{2}\right|_{y=1}=0
\end{gathered}
$$


Для нахождения соответствуюших значений $\beta$ рассмотрим полный набор собственных значений и собственных функций краевой задачи (2.47):

$$
\begin{aligned}
\lambda_{k}^{1}=i \sigma_{0} \pi^{2}\left[(\beta+k)^{2}-1\right], & e_{k}^{1}(y)=\operatorname{colon}(\cos k \pi y, 0, \sin k \pi y, 0), \\
k=0,1,2, \ldots ; & \\
\lambda_{k}^{2}=-i \sigma_{0} \pi^{2}\left[(\beta+k)^{2}-1\right], & e_{k}^{2}(y)=\operatorname{colon}(0, \cos k \pi y, 0, \sin k \pi y), \\
k=0,1,2, \ldots ; & \\
\lambda_{k}^{3}=i \sigma_{0} \pi^{2}\left[(\beta-k)^{2}-1\right], & e_{k}^{3}(y)=\operatorname{colon}(\cos k \pi y, 0,-\sin k \pi y, 0), \\
k=1,2, \ldots ; & \\
\lambda_{k}^{4}=-i \sigma_{0} \pi^{2}\left[(\beta-k)^{2}-1\right], & e_{k}^{4}(y)=\operatorname{colon}(0, \cos k \pi y, 0,-\sin k \pi y), \\
k=1,2, \ldots &
\end{aligned}
$$

Несложная проверка (с учетом формул $(2.48))$ показывает, что при $(k, j) \neq(0,1)$ уравнения

$$
\lambda_{k}^{j}(\beta)-\lambda_{0}(\beta)=0
$$

имеют следуюшие решения:

$$
\begin{array}{rlrl}
\beta & =1 & \text { при } & (k, j)=(0,2),(2,3),(2,4) ; \\
\beta=\frac{\sqrt{3}+1}{2} & \text { при } & (k, j)=(1,4) ; \\
\beta=\frac{\sqrt{3}-1}{2} & \text { при } & (k, j)=(1,2) ; \\
\beta=\frac{m}{2}, m \geqslant 1, \quad m \neq 2, & \text { при } & (k, j)=(m, 3) .
\end{array}
$$

Более того, все эти корни простые, за исключением корня $\beta=1$ при $(k, j)=(2,4)$, для которого выполняются условия

$$
\left.\frac{d}{d \beta}\left[\lambda_{2}^{4}(\beta)-\lambda_{0}(\beta)\right]\right|_{\beta=1}=0,\left.\quad \frac{d^{2}}{d \beta^{2}}\left[\lambda_{2}^{4}(\beta)-\lambda_{0}(\beta)\right]\right|_{\beta=1} \neq 0 .
$$

Итак, собственное значение $\lambda_{0}(\beta)$ (см. (2.42)) является: простым при

$$
\beta \neq \frac{m}{2}, \quad m=1,2, \ldots, \quad \beta \neq \frac{\sqrt{3} \pm 1}{2}
$$

четырехкратным при $\beta=1$ с собственными функциями

$$
\begin{aligned}
& \theta_{1}(y)=\operatorname{colon}(1,0,0,0), \\
& \theta_{2}(y)=\operatorname{colon}(0,1,0,0), \\
& \theta_{3}(y)=\operatorname{colon}(\cos 2 \pi y, 0,-\sin 2 \pi y, 0), \\
& \theta_{4}(y)=\operatorname{colon}(0, \cos 2 \pi y, 0,-\sin 2 \pi y) ;
\end{aligned}
$$


двукратным при $\beta=(\sqrt{3} \pm 1) / 2$ соответственно с собственными функциями

$$
\theta_{1}(y)=\operatorname{colon}(1,0,0,0), \quad \theta_{2}(y)=\operatorname{colon}(0, \cos \pi y, 0,-\sin \pi y)
$$

или

$$
\theta_{1}(y)=\operatorname{colon}(1,0,0,0), \quad \theta_{2}(y)=\operatorname{colon}(0, \cos \pi y, 0, \sin \pi y) ;
$$

двукратным при $\beta=m / 2, m \geqslant 1, m \neq 2$, с собственными функциями

$$
\theta_{1}(y)=\operatorname{colon}(1,0,0,0), \quad \theta_{2}(y)=\operatorname{colon}(\cos m \pi y, 0,-\sin m \pi y, 0) .
$$

При условиях (2.50), т.е. в простейшей ситуации, асимптотику собственного значения (2.46) и отвечающей ему собственной функции будем искать в виде

$$
\lambda=\lambda_{0}(\beta)+\alpha \lambda_{1}(\beta)+\cdots, \quad \eta=\theta_{0}(y)+\alpha \theta_{1}(y, \beta)+\cdots
$$

где $\lambda_{0}(\beta)$ такое, как в $(2.42)$, а $\theta_{0}(y)=\operatorname{colon}(1,0,0,0)$. Подставляя эти формулы в соотношения (2.44) и приравнивая коэффициенты при $\alpha$, для поправки $\theta_{1}(y, \beta)$ получаем краевую задачу

$$
S(\beta) \theta_{1}=\lambda_{1} \theta_{0}(y)-B_{2}(y) \theta_{0}(y)
$$

где $S(\beta)$ - дифференшиальный оператор, порожденный краевой задачей (2.47) при $\lambda=\lambda_{0}(\beta)$. Стандартный анализ задачи $(2.56)$ приводит к равенствам

$$
\begin{aligned}
\lambda_{1}(\beta) & =-\frac{\delta}{3}\left(1+i c_{0}\right), \quad \theta_{1}(y, \beta)=\frac{\delta\left(1+i c_{0}\right)}{3}\left[\frac{2 e_{0}^{2}(y)}{\bar{\lambda}_{0}(\beta)-\lambda_{0}(\beta)}\right. \\
& \left.+\frac{2 e_{2}^{1}(y)}{\lambda_{2}^{1}(\beta)-\lambda_{0}(\beta)}+\frac{e_{2}^{2}(y)}{\lambda_{2}^{2}(\beta)-\lambda_{0}(\beta)}+\frac{2 e_{2}^{3}(y)}{\lambda_{2}^{3}(\beta)-\lambda_{0}(\beta)}+\frac{e_{2}^{4}(y)}{\lambda_{2}^{4}(\beta)-\lambda_{0}(\beta)}\right]
\end{aligned}
$$

где, напомним, $\lambda_{k}^{j}(\beta), e_{k}^{j}(y)$ задаются формулами $(2.48)$.

Для придания асимптотическим равенствам (2.55) строгого смысла фиксируем произвольное число $\Delta>0$ и рассмотрим множество значений $\beta \in F=F(\alpha, \Delta)$, где

$$
\begin{gathered}
F=\mathbb{R}^{+} \backslash\left\{(1-\Delta \sqrt{\alpha}, 1+\Delta \sqrt{\alpha}) \cup\left(\beta_{-}-\Delta \alpha, \beta_{-}+\Delta \alpha\right)\right. \\
\left.\cup\left(\beta_{+}-\Delta \alpha, \beta_{+}+\Delta \alpha\right) \cup \sum_{m=1, m \neq 2}^{\infty}\left(\frac{m}{2}-\Delta \alpha, \frac{m}{2}+\Delta \alpha\right)\right\} ; \\
\mathbb{R}^{+}=\{\beta: \beta \geqslant 0\}, \quad \beta_{ \pm}=\frac{\sqrt{3} \pm 1}{2}
\end{gathered}
$$

(отход порядка $\sqrt{\alpha}$ от особенности $\beta=1$ продиктован дополнительньм вырождением (2.49)). Справедливо следуюшие утверждение. 
ЛЕмма 2.5. По любому фиксированному числу $\Delta>0$ можнн указать такое число $\alpha_{0}=\alpha_{0}(\Delta)$, что при $0 \leqslant \alpha \leqslant \alpha_{0}, \quad \beta \in F(\alpha, \Delta)$ для собственного значения (2.46) краевой задачи (2.44) справедливо асимптотическое представление

$$
\lambda(\alpha, \beta)=\lambda_{0}(\beta)+\alpha \lambda_{1}+\alpha \psi(\alpha, \beta), \quad|\psi| \leqslant \frac{M}{\Delta},
$$

где постоянная $M>0$ не зависит от $\alpha, \beta, \Delta$.

ДокАЗАТЕЛЬСТво. При $\beta \in F(\alpha, \Delta)$ положим в краевой задаче $(2.44)$ (см. $(2.55))$

$$
\lambda=\lambda_{0}(\beta)+\alpha \lambda_{1}+\alpha \psi(\alpha, \beta), \quad \eta=\theta_{0}(y)+\alpha \theta_{1}(y, \beta)+R(y, \alpha, \beta, \psi) .
$$

В итоге получим краевую задачу

$$
S(\beta) R=\Psi, \quad \Psi=-\alpha B_{2}(y)\left(\alpha \theta_{1}+R\right)+\alpha \lambda_{1}\left(\alpha \theta_{1}+R\right)+\alpha \psi\left(\theta_{0}+\alpha \theta_{1}+R\right) .
$$

Изучим вопрос об обратимости оператора $S(\beta)$ в подпространстве $L_{2}^{0} \subset$ $L_{2}\left([0,1] ; \mathbb{C}^{4}\right)$, ортогональном функции $\theta_{0}(y)$. Метод Фурье по собственным функциям $(2.48)$ приводит к равенствам $\left(f(x)\right.$ - произвольная функция из $\left.L_{2}^{0}\right)$

$$
\begin{gathered}
f(x)=f_{0} e_{0}^{2}(y)+\sum_{k=1}^{\infty} \sum_{j=1}^{4} f_{k}^{j} e_{k}^{j}(y) \\
S^{-1}(\beta) f(x)=\frac{f_{0}}{\bar{\lambda}_{0}(\beta)-\lambda_{0}(\beta)} e_{0}^{2}(y)+\sum_{k=1}^{\infty} \sum_{j=1}^{4} \frac{f_{k}^{j}}{\lambda_{k}^{j}(\beta)-\lambda_{0}(\beta)} e_{k}^{j}(y) .
\end{gathered}
$$

Учитывая здесь отмеченные выше свойства знаменателей $\lambda_{k}^{j}(\beta)-\lambda_{0}(\beta)$ и структуру множества $F$, приходим к оценке

$$
\left\|S^{-1}(\beta)\right\|_{L_{2}^{0} \rightarrow L_{2}^{0}} \leqslant \frac{M}{\alpha \Delta} .
$$

Дальнейший способ действий стандартен (см. аналогичное место в доказательстве леммы 2.2): сначала переходим от (2.59) к вспомогательному уравнению

$$
S(\beta) R=\Psi-\int_{0}^{1}\left(\Psi, \theta_{0}(y)\right) d y \cdot \theta_{0}(y)
$$

в $L_{2}^{0}$, затем обрашаем оператор $S(\beta)$ и, наконец, к получившемуся интегральному уравнению в $L_{2}^{0}$ применяем принцип сжимающих отображений, справедливость которого гарантируют неравенство (2.60) и оценка

$$
\left\|\theta_{1}\right\|_{L_{2}\left([0,1] ; \mathbb{C}^{4}\right)} \leqslant \frac{M}{\alpha \Delta}
$$

(вытекаюшая из явного вида $(2.57)$ функции $\left.\theta_{1}\right)$. В результате находим функцию $R(y, \alpha, \beta, \psi)$, удовлетворяюшую неравенству

$$
\|R\|_{L_{2}^{0}}+\left\|\frac{\partial R}{\partial \psi}\right\|_{L_{2}^{0}} \leqslant \frac{M}{\Delta^{2}} .
$$

Зануляя теперь поправку к неоднородности $\Psi$ в уравнении $(2.61)$ и учитывая оценку (2.62), находим функцию $\psi(\alpha, \beta)$, для которой $|\psi| \leqslant M / \Delta$. Лемма доказана. 
2.7. Построим асимптотику собственного значения (2.46) при $\beta \in \mathbb{R}^{+} \backslash F$, т.е. в окрестности так называемых пространственных резонансов.

Начнем со случая

$$
\beta \in \bigcup_{m=1, m \neq 2}^{\infty}\left(\frac{m}{2}-\Delta \alpha, \frac{m}{2}+\Delta \alpha\right) .
$$

Напомним, что в конечном итоге нас интересуют дискретные значения $k / n$ параметра $\beta$, лежашие друг от друга на расстоянии $1 / n=\sqrt{\alpha}$. С учетом этого обстоятельства множество (2.63) сужается до точек

$$
\beta=\frac{m}{2}, \quad m \geqslant 1, \quad m \neq 2 .
$$

Но при этих условиях, когда собственное значение $\lambda_{0}(\beta)$ краевой задачи $(2.47)$ двукратно, описанный в предыдушем пункте алгоритм естественным образом изменяется.

В рассматриваемом случае перейдем от краевой задачи (2.44) к матричной краевой задаче

$$
\begin{gathered}
S\left(\frac{m}{2}\right) V+\alpha B_{2}(y) V=V H \\
\left.\frac{d}{d y} P_{1} V\right|_{y=0}=\left.\frac{d}{d y} P_{1} V\right|_{y=1}=\left.P_{2} V\right|_{y=0}=\left.P_{2} V\right|_{y=1}=0
\end{gathered}
$$

где матрицы $V$ и $H$ имеют размеры $4 \times 2$ и $2 \times 2$ соответственно,

$$
P_{1}=\operatorname{diag}\{I, 0\}, \quad P_{2}=\operatorname{diag}\{0, I\},
$$

а 0 и $I$ - нулевая и единичная квадратные матрицы 2-го порядка. Подставляя, далее, в задачу (2.64) ряды

$$
V=V_{0}(y)+\alpha V_{1}(y)+\cdots, \quad H=\alpha H_{1}+\cdots,
$$

где $V_{0}(y)$ - матрица размера $4 \times 2$, столбцами которой служат собственные функции $\theta_{1}(y), \theta_{2}(y)$ из $(2.54)$, и приравнивая коэффициенты при $\alpha$, для $V_{1}$ получаем краевую задачу для уравнения (граничные условия для краткости опускаем)

$$
S\left(\frac{m}{2}\right) V_{1}=V_{0}(y) H_{1}-B_{2}(y) V_{0}(y) .
$$

Из условий ее разрешимости сначала находим

$$
H_{1}=\int_{0}^{1} V_{0}^{*}(y) B_{2}(y) V_{0}(y) d y=-\frac{\delta}{3}\left(1+i c_{0}\right) I
$$

а затем, решая уравнение (2.66), определяем и саму матрицу $V_{1}$.

Для придания равенствам (2.65) строгого смысла введем в рассмотрение банахово пространство $J$ матрищ размера $4 \times 2$

$$
V(y)=\left[v_{1}(y), v_{2}(y)\right], \quad v_{j}(y) \in L_{2}\left([0,1] ; \mathbb{C}^{4}\right), \quad j=1,2,
$$

с нормой

$$
\|V\|_{J}=\left\|v_{1}\right\|_{L_{2}}+\left\|v_{2}\right\|_{L_{2}} .
$$

Оказывается справедливым следуюшее утверждение. 
Лемма 2.6. Найдется такое достаточно малое число $\alpha_{0}>0$, что при $0 \leqslant \alpha \leqslant \alpha_{0}$ и при каждом натуральном $m \geqslant 1, m \neq 2$, существует пара матрии $V(y, \alpha, m), H(\alpha, m)$, удовлетворяющая краевой задаче (2.64) и допускающая асимптотику

$$
\begin{gathered}
V(y, \alpha, m)=V_{0}(y)+\alpha V_{1}(y)+\alpha^{2} V_{2}(y, \alpha, m), \\
H(\alpha, m)=\alpha H_{1}+\alpha^{2} H_{2}(\alpha, m), \quad\left\|V_{2}\right\|_{J} \leqslant M, \quad\left\|H_{2}\right\| \leqslant M,
\end{gathered}
$$

где константа $M>0$ не зависит от $\alpha, m$.

ДоКАЗАТЕЛЬСТВО леммы практически дословно повторяет обоснование леммы 2.5 и базируется на оценке

$$
\left\|S^{-1}\left(\frac{m}{2}\right)\right\|_{J_{0} \rightarrow J_{0}} \leqslant M
$$

где $J_{0}$ - подпространство $J$, состоящее из таких матрищ $V(y)$, что

$$
\int_{0}^{1} V_{0}^{*}(y) V(y) d y=0
$$

При рассмотрении остальных пространственных резонансов, т.е. не вошедших в $F$ отрезков

$$
\beta_{ \pm}-\Delta \alpha \leqslant \beta \leqslant \beta_{ \pm}+\Delta \alpha, \quad 1-\Delta \sqrt{\alpha} \leqslant \beta \leqslant 1+\Delta \sqrt{\alpha},
$$

изложим только алгоритмическую часть, опуская стандартные обоснования.

При $\beta=\beta_{ \pm}+b \alpha,|b| \leqslant \Delta$, исследованию подлежит матричная краевая задача (аналогичная (2.64))

$$
\begin{gathered}
S(\beta) V+\alpha B_{2}(y) V=V H \\
\left.\frac{d}{d y} P_{1} V\right|_{y=0}=\left.\frac{d}{d y} P_{1} V\right|_{y=1}=\left.P_{2} V\right|_{y=0}=\left.P_{2} V\right|_{y=1}=0 .
\end{gathered}
$$

Подставляя сюда ряды (2.65), где теперь $\theta_{1}(y), \theta_{2}(y)$ - собственные функции $(2.52)$ или (2.53), и действуя описанным вьше образом, убеждаемся в том, что

$$
H_{1}=\operatorname{diag}\left\{-\frac{\delta}{3}\left(1+i c_{0}\right),-\frac{\delta}{3}\left(1-i c_{0}\right) \pm 2 i \sigma_{0} \pi^{2} b-4 i \sigma_{0} \pi^{2} \beta_{ \pm} b\right\}
$$

При $\beta=1+b \sqrt{\alpha},|b| \leqslant \Delta$, снова обратимся к краевой задаче (2.68), считая $V$ и $H$ квадратными матрицами 4-го порядка. Подставляя в нее ряды

$$
V=V_{0}(y)+\sqrt{\alpha} V_{1}(y)+\alpha V_{2}(y)+\cdots, \quad H=\sqrt{\alpha} H_{1}+\alpha H_{2}+\cdots,
$$


где $V_{0}(y)$ - квадратная матрица 4-го порядка, столбцами которой служат собственные функции (2.51), и приравнивая коэффициенты при $\sqrt{\alpha}$ и $\alpha$, последовательно находим

$$
\begin{gathered}
H_{1}=\operatorname{diag}\left\{0,-4 i \pi^{2} \sigma_{0} b,-4 i \pi^{2} \sigma_{0} b, 0\right\}, \quad V_{1}=0 ; \\
H_{2}=\left(\begin{array}{cc}
H_{2,1} & H_{2,2} \\
H_{2,2} & H_{2,1}
\end{array}\right), \\
H_{2,1}=\left(\begin{array}{cc}
p_{0} & 2 p_{0} \\
2 \bar{p}_{0} & \bar{p}_{0}-2 i \pi^{2} \sigma_{0} b^{2}
\end{array}\right), \quad H_{2,2}=\left(\begin{array}{cc}
2 p_{0} & p_{0} \\
\bar{p}_{0} & 2 \bar{p}_{0}
\end{array}\right), \\
p_{0}=-\frac{\delta}{3}\left(1+i c_{0}\right) .
\end{gathered}
$$

Проведенные построения полностью решают проблему устойчивости циклов (2.40). Действительно, увеличивая подходящим образом параметр $\Delta$ в определении множества $F$, убеждаемся, что $\operatorname{Re} \lambda(\alpha, \beta)<0$ (см. (2.58)). Далее, собственные значения матриц (2.67), (2.69) также имеют отрицательные действительные части. Таким образом, проблема сводится к анализу расположения собственных значений матрицы (см. (2.70))

$$
H_{1}+\sqrt{\alpha} H_{2},
$$

где параметр $b$ принимает только целые значения $0, \pm 1, \pm 2, \ldots$ из отрезка $-\Delta \leqslant$ $b \leqslant \Delta$ (так как, напомним, параметр $\beta=k / n$ дискретен).

При $b=0$ собственными значениями матрицы $(2.71)$ являются

$$
\lambda_{1}=\lambda_{2}=0, \quad \lambda_{3}=-2 \delta, \quad \lambda_{4}=\frac{2 \delta}{3} .
$$

Заметим, однако, что после перехода к исходной переменной $w$ (см. (2.43)) двум первым из них будет отвечать только одна собственная функция, а числу $\lambda_{4}-$ нулевая собственная функция. Тем самым собственные значения $\lambda_{2}$ и $\lambda_{4}$ фиктивные, их появление обусловлено способом перехода от краевой задачи (2.41) к вспомогательной задаче (2.44).

Исследование матрищы (2.71) при $b=m, m= \pm 1, \pm 2, \ldots$, стандартно: поправки порядка $\sqrt{\alpha}$ к ее собственным значениям $\lambda_{1}=\lambda_{2}=0, \lambda_{3}=\lambda_{4}=-4 i \pi^{2} \sigma_{0} m$ определяются как собственные значения матриц

$$
\left(\begin{array}{cc}
p_{0} & p_{0} \\
\bar{p}_{0} & \bar{p}_{0}-2 i \pi^{2} m^{2} \sigma_{0}
\end{array}\right), \quad\left(\begin{array}{cc}
\bar{p}_{0}-2 i \pi^{2} m^{2} \sigma_{0} & \bar{p}_{0} \\
p_{0} & p_{0}
\end{array}\right)
$$

соответственно. Их анализ приводит к следуюшему утверждению.

Лемма 2.7. Автомодельнье иикль (2.40) краевой задачи (2.11) экспоненииально орбитально устойчивы (неустойчивь) при

$$
\delta-\frac{3}{2} \pi^{2} \frac{\sigma_{0}}{c_{0}}<0 \quad(>0)
$$

Объединяя леммы $2.1,2.3,2.4,2.7$, убеждаемся в непустоте множеств $\Omega_{n}$ (см. п. 1.2) при любом натуральном $n$. Теорема 1.2 полностью доказана 


\section{§ 3. Заключительные замечания}

3.1. Обратим внимание на тот факт, что при всех

$$
\gamma>\gamma_{0}=\frac{\varkappa_{1}-\frac{\pi}{2} \varkappa_{2}}{\varkappa_{1}^{2}+\varkappa_{2}^{2}}
$$

где, напомним, $\varkappa_{1}$ и $\varkappa_{2}$ определены в п. 1.2, нормальная форма $(2.1)$ имеет пространственно однородный (т.е. не зависящий от $x$ ) цикл

$$
\xi(\tau)=\xi_{0} \exp \left(i \omega_{0} \tau\right), \quad \xi_{0}=\sqrt{\gamma-\gamma_{0}}, \quad \omega_{0}=-c_{0} \xi_{0}^{2}-\operatorname{Im}\left[\frac{1-i \frac{\pi}{2}}{\varkappa}\right]
$$

здесь $\varkappa=\varkappa_{1}+i \varkappa_{2}$. Рассматривая, далее, отвечаюшую ему краевую задачу (2.13) и применяя к ней метод Фурье по системе функщий $\cos n \pi x, n=0,1,2, \ldots$, убеждаемся, что за устойчивость цикла (3.2) отвечает спектр матрищ

$$
\left(\begin{array}{ll}
b_{n, 1} & b_{n, 2} \\
\bar{b}_{n, 2} & \bar{b}_{n, 1}
\end{array}\right), \quad n=0,1,2, \ldots
$$

где

$$
b_{n, 1}=-\left(1+i c_{0}\right) \xi_{0}^{2}-\left(1-i \frac{\pi}{2}\right)\left[\frac{1}{D \pi^{2} n^{2}+\varkappa}-\frac{1}{\varkappa}\right], \quad b_{n, 2}=-\left(1+i c_{0}\right) \xi_{0}^{2}
$$

Для формулировки следующего утверждения потребуются функции

$$
\begin{gathered}
w=\Phi(z)=z \frac{\varphi_{1}+\varphi_{2} z}{\varkappa_{1}^{2}+\varkappa_{2}^{2}}\left[\left(\varkappa_{1}+z\right)^{2}+\varkappa_{2}^{2}\right], \quad z \geqslant 0 \\
z=\Psi(w)=\frac{2 \psi_{1} w}{1+\frac{\pi^{2}}{4}+2 \psi_{2} w}, \quad w \geqslant 0
\end{gathered}
$$

где

$$
\begin{aligned}
& \varphi_{1}=\varkappa_{1}^{2}-\varkappa_{2}^{2}-\pi \varkappa_{1} \varkappa_{2}, \quad \varphi_{2}=\varkappa_{1}-\frac{\pi}{2} \varkappa_{2} ; \\
& \psi_{1}=\left(\frac{\pi}{2} c_{0}-1\right)\left(\varkappa_{2}^{2}-\varkappa_{1}^{2}\right)-2\left(\frac{\pi}{2}+c_{0}\right) \varkappa_{1} \varkappa_{2}, \\
& \psi_{2}=\left(\frac{\pi}{2} c_{0}-1\right) \varkappa_{1}+\left(\frac{\pi}{2}+c_{0}\right) \varkappa_{2} .
\end{aligned}
$$

Введем также в рассмотрение на плоскости $(w, z)$ области

$$
\begin{gathered}
U_{1}=\{(z, w): z, w>0, w>\Phi(z), z>\Psi(w)\}, \\
U_{2}=K \backslash \bar{U}_{1}, \quad K=\{(z, w): z>0, w>0\} .
\end{gathered}
$$



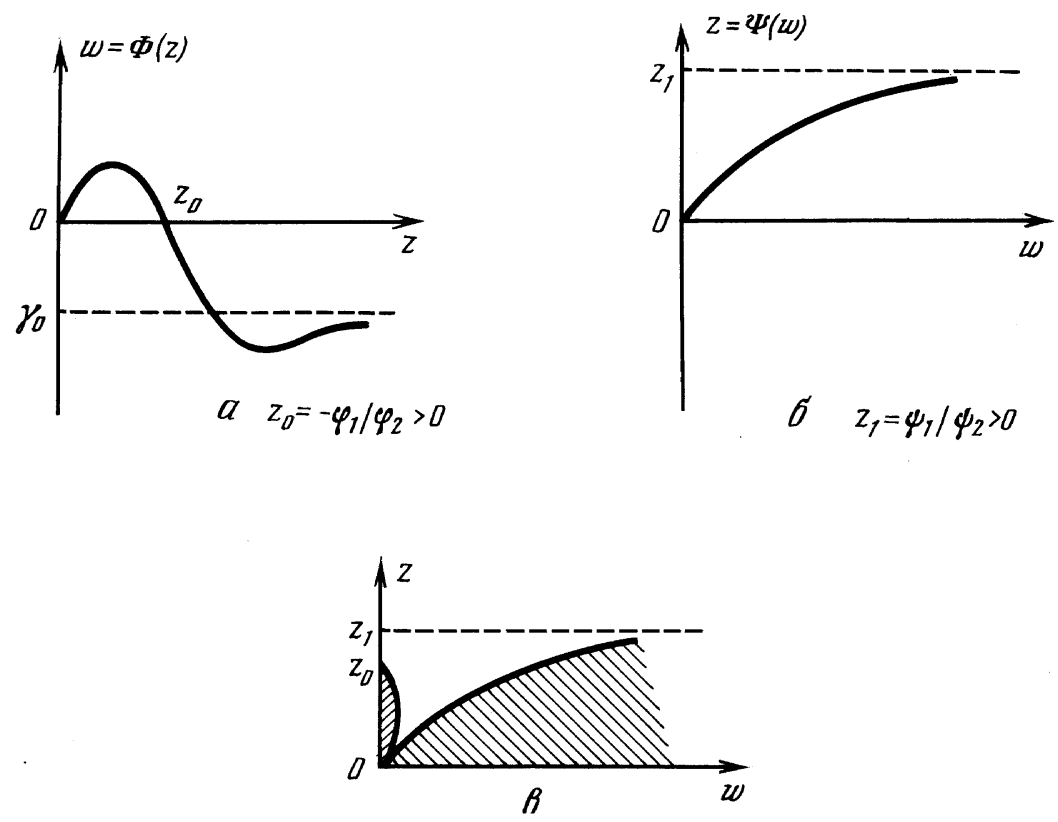

Рис. 2

ЛЕмма 3.1. Предположим, что параметры краевой задачи (2.1), кроме неравенств (1.7) и (3.1), удовлетворяют еще условиям

$$
\varphi_{1}>0, \quad \varphi_{2}<0, \quad \psi_{1}>0, \quad \psi_{2}>0
$$

Тогда чикл (3.2) орбитально әкспоненциально устойчив, если при каждом $n=1,2, \ldots$ справедливы включения

$$
\left(D \pi^{2} n^{2}, \xi_{0}^{2}\right) \in U_{1},
$$

и неустойчив, если для некоторого $n_{0} \geqslant 1$ имеет место включение

$$
\left(D \pi^{2} n_{0}^{2}, \xi_{0}^{2}\right) \in U_{2} .
$$

ДокАЗАТЕЛЬСТво леммы основано на несложном анализе матрищ (3.3), который здесь опустим. Отметим лишь, что если точка $\left(D \pi^{2} n^{2}, \xi_{0}^{2}\right)$ попадает на часть границы области $U_{1}$, задаваемую равенством $w=\Phi(z)$ (или $z=\Psi(w)$ ), то соответствуюшая матрица (3.3) имеет нулевое (или чисто мнимое) собственное значение.

При условиях (3.6) графики функций (3.4) имеют вид, показанньй на рис. $2, a$ и 2,6 , а типичный характер расположения областей (3.5) представлен на рис. $2, \theta$ 
(область $U_{2}$ заштрихована). Заметим также, что значениям параметров (1.5) соответствует набор параметров

$$
\begin{gathered}
\varkappa_{1}=-0.551417 \ldots, \quad \varkappa_{2}=1.8796049 \ldots, \\
0.0008 \leqslant D \leqslant 0.004, \quad 1.6022 \ldots \leqslant \gamma \leqslant 33.52306 \ldots,
\end{gathered}
$$

при которых цикл (3.2) неустойчив; в данном случае выполняются неравенства (3.6), причем (см. рис. $2, \boldsymbol{\theta})$

$$
z_{0}=0,007774 \ldots, \quad z_{1}=1,8570469 \ldots
$$

область $U_{2}$ имеет показанный на рис. 2, в вид и содержит некоторые из точек $\left(D \pi^{2} n^{2}, \xi_{0}^{2}\right)$.

Для прояснения смысла леммы 3.1 рассмотрим отвечающую краевой задаче (1.8) точечную модель, т.е. систему

$$
\dot{u}=-\left(\frac{\pi}{2}+\gamma \varepsilon\right)[\varepsilon v+u(t-1)](1+u), \quad \dot{v}=r[u-v(t-h)](1+v) .
$$

Циклу (3.2) этой системы соответствует экспоненциально орбитально устойчивый цикл

$$
u=u_{0}(t, \varepsilon), \quad v=v_{0}(t, \varepsilon)
$$

с асимптотикой (1.11), (3.2), существующий в силу бифуркационной теоремы Андронова-Хопфа [10]. Приведенная же лемма 3.1 решает проблему устойчивости цикла (3.8) в рамках распределенной модели (1.8).

3.2. Из содержащихся в $\S 2$ результатов вытекают следуюшие характерные особенности динамики краевой задачи (1.8) при увеличении параметра $\gamma$.

ВТОРИЧнАЯ БУФЕРНОСТЬ. При увеличении $\gamma$ неустойчивые пространственно неоднородные циклы, ответвляюшиеся от нулевого состояния равновесия в результате вторичных бифуркаций Андронова-Хопфа, становятся устойчивыми, возрастая по амплитуде. Отметим, что именно такие циклы конструировались при доказательстве теоремы 1.2. Более того, показано, что при подходяшем уменьшении коэффициента диффузии таких устойчивых циклов может быть любое конечное число (но не счетное, как в случае краевой задачи (2.11)).

УСЛОжнЕнИЕ динАмИКИ. При дальнейшем увеличении $\gamma$ все пространственно неоднородные циклы, о которых говорилось в $\S 1,2$, теряют устойчивость колебательным образом (см. (2.72)). Данное обстоятельство согласуется с результатом монографии [16] о том, что усложнение динамики при увеличении мальтузианского коэффициента линейного роста жертвы происходит уже в рамках точечной модели (системы обыкновенных дифференциальных уравнений, получающейся из уравнений $(1.1),(1.2)$ при $D=0)$. Приведенные вьше результаты показывают, что в рамках распределенной модели этот процесс идет значительно быстрее. В самом деле, в распределенной модели (1.8) имеет место диффузионная буферность, а в отвечающей ей точечной модели (3.7) - лишь единственный устойчивый цикл (3.8). 
ЭЛЕМЕНТЫ САМООРГАНИЗАЦИИ. УстойчивЫе Пространственно неоднородные циклы, доставляемые теоремой 1.2 , могут сосушествовать с устойчивым однородным циклом. Для доказательства достаточно заметить, что однородный цикл

$$
\xi(\tau)=\xi_{0} \exp \left(i \omega_{0} \tau\right), \quad \xi_{0}=\sqrt{\delta}, \quad \omega_{0}=-c_{0} \delta
$$

краевой задачи (2.11) экспоненциально орбитально устойчив (неустойчив) при

$$
\delta-\frac{\pi}{2} \frac{\sigma_{0}}{c_{0}}<0 \quad(>0)
$$

и снова воспользоваться леммами $2.1,2.3,2.4,2.7$.

Итак, может возникать достаточно странная на первый взгляд ситуация: однородный цикл устойчив и, казалось бы, нет необходимости в сушествовании других устойчивых циклов. Однако они существуют, причем в достаточно большом количестве, и имеют лучшие биологические характеристики - меньше размах колебаний хищника и больше его средняя численность (в рамках изложенной в $§ 2$ асимптотической теории эти эффекты незначительны, но хорошо видны при численном эксперименте [1]). Тем самым можно говорить об элементах самоорганизации у хищника: он искусственно (за счет миграций) создает себе неоднородную среду обитания и повышает свой "уровень жизни”.

В заключение отметим, что в принципе все перечисленные выше особенности динамики были выявлены в работах [2]-[5] на модельной параболической системе с мальми коэффициентами диффузии. Однако перенос соответствуюших результатов на исходную задачу (1.1)-(1.3), как выяснилось, отнюдь не тривиален. В частности, в статьях [2]-[5] не использовался метод бесконечномерной нормализации и нет аналога интегро-дифференциальной краевой задачи (1.12).

\section{Список литературы}

1. Захаров А.А., Колесов Ю.С. Пространственно неоднородные режимы в задаче хищник-жертва // Нелинейные колебания и экология. Ярославль, 1984. С. 3-15.

2. Колесов А. Ю. Существование асимптотически большого числа устойчивых бегущих волн в одной трехмерной системе параболических уравнений // Методы качественной теории дифференциальных уравнений. Горький, 1986. С. 57-66.

3. Колесов А. Ю. Автоколебания сингулярно возмущенных параболических систем первой степени негрубости // Матем. заметки. 1991. Т. 49. № 5. С. 62-69.

4. Колесов А. Ю. Бифуркация периодических решений сингулярно возмущенных параболических систем первой степени негрубости // Укр. матем. журн. 1990. Т. 42 . № 8 . C. $1037-1042$.

5. Колесов А. Ю. Теоретическое объяснение явления диффузионной буферности // Моделирование динамики популяций. Н. Новгород, 1990. С. 35-38.

6. Мищенко Е.Ф., Колесов Ю.С., Колесов А. Ю., Розов Н.Х. Периодические движения и бифуркационные процессы в сингулярно возмущенных системах. М.: Физматлит, 1995.

7. Колесов Ю.С. Математические модели экологии // Исследования по устойчивости и теории колебаний. Ярославль, 1979. С. 3-40. 
8. Ильин A. М., Калашников A.C., Олейник O. А. Линейные уравнения второго порядка параболического типа // УМН. 1962. Т. 17. № 3. С. 3-146.

9. Ладыженская О.А., Солонников В. А., Уральцева Н. Н. Линейные и квазилинейные уравнения параболического типа. М.: Наука, 1967.

10. Колесов Ю. С., Швитра Д. И. Автоколебания в системах с запаздыванием. Вильнюс: Мокслас, 1979.

11. Хартман . Обыкновенные дифференциальные уравнения. М.: Мир, 1970.

12. Куликов A. Н. О гладких инвариантных многообразиях полугруппы нелинейных операторов в банаховом пространстве // Исследования по устойчивости и теории колебаний. Ярославль, 1976. С. 114-129.

13. Ладыженская О.А., Уральцева H. Н. Линейные и квазилинейные уравнения эллиптического типа. М.: Наука, 1964.

14. Наймарк М. А. Линейные дифференциальные операторы. М.: Наука, 1969.

15. Като Т. Теория возмущений линейных операторов. М.: Мир, 1972.

16. Колесов А. Ю., Колесов Ю. С. Релаксационные колебания в математических моделях экологии // Тр. МИРАН. 1993. Т. 199. С. 3-125.

Поступило в редакцию 4.XI.1996 